\title{
Stationary Eddy Response to Surface Boundary Forcing: Idealized GCM Experiments*
}

\author{
Masaru InAtsu and Hitoshi Mukougawa \\ Graduate School of Environmental Earth Science, Hokkaido University, Sapporo, Japan \\ SHANG-PING XIE \\ International Pacific Research Center/SOEST and Department of Meteorology, University of Hawaii, Honolulu, Hawaii
}

(Manuscript received 31 October 2000, in final form 21 November 2001)

\begin{abstract}
A set of atmospheric general circulation model (AGCM) experiments under idealized conditions is performed to investigate atmospheric response to surface boundary forcing by extratropical land-sea contrast, large-scale orography, and tropical sea surface temperature (SST) distribution. Stationary eddies forced by the extratropical land-sea distribution are strongest in high latitudes, but their amplitudes are modest and comparable to internal chaotic variability. By contrast, the stationary eddy response to zonal variations in tropical SST is strong and robust in both the subtropics and midlatitudes. While these SST-forced stationary waves are trapped within the troposphere, those induced by orography show a strong vertical propagation into the stratosphere. Analysis of transient eddies indicates that orography is effective in generating a zonally localized storm track while extratropical land-sea contrast has little effect on the zonal variation of upper-level storm activity.

A vorticity budget analysis is carried out to understand tropical SST forcing mechanism to set up extratropical stationary eddies. In the subtropics, the dominant balance is reached between the vortex stretching and zonal advection. North of the tropical warm water pool, a subtropical anticyclone forms in the upper troposphere in response to the divergence of the locally enhanced Hadley circulation. The authors further show that this subtropical response to tropical SST variations has nonlinear characteristics in both its amplitude and zonal phase.
\end{abstract}

\section{Introduction}

The wintertime atmospheric flow in the Northern Hemisphere displays large zonal asymmetry. The upper tropospheric zonal wind has well-defined maxima called subtropical westerly jet cores over the east coast of Asia and North America (Blackmon et al. 1977), in association with extratropical wave patterns in the time-mean height field (Lau 1979; Wallace 1983). For example, zonal wind speed at $250 \mathrm{hPa}$ exceeds $70 \mathrm{~m} \mathrm{~s}^{-1}$ over Japan, straddling a low pressure center over eastern $\mathrm{Si}$ beria and a high over the Philippines. In the lower troposphere, temperature varies strongly in the zonal direction: very cold over the continents but relatively warm over the oceans. These geographically fixed patterns appear not only in the time-mean fields but also

\footnotetext{
* International Pacific Research Center Contribution Number 144, and School of Ocean and Earth Science and Technology Contribution Number 5916.
}

Corresponding author address: Mr. Masaru Inatsu, Graduate School of Environmental Earth Science, Hokkaido University, North 10 West 5, Sapporo 0600810, Japan.

E-mail: inaz@ees.hokudai.ac.jp in synoptic variability. Synoptic height fluctuations are stronger over the Pacific and Atlantic in zonally localized bands called storm tracks (Blackmon 1976).

Extensive literature exists on the mechanisms for developing zonal asymmetry in the Northern Hemisphere winter climate. The forcing lies ultimately on earth's surface: large-scale mountains, zonal variations in sea surface temperature (SST), and land-sea distribution. Their relative importance for stationary eddies has been mainly investigated by linear models. Charney and Eliassen (1949) and Smagorinsky (1953) pioneered the study of the linear response to orographic and extratropical thermal forcing, while tropical thermal forcing is emphasized by Simmons (1982). Hoskins and Karoly (1981) built a theoretical framework for studying this problem on the sphere. They examined the response of a linear model to various idealized orographic and thermal forcings, and noted that the response to these two forcings differs qualitatively: tropical heating induces local ascending motion, extratropical heating causes the northward heat transport, and orography induces a high sea level pressure (SLP) around the summit. Nigam et al. $(1986,1988)$ studied the response of a linear model to forcing derived from two atmospheric general circulation model (AGCM) 
simulations with and without mountains. They found that extratropical forcing is more important than the tropical one and that the quantitative estimation is somewhat sensitive to the damping imposed in the model Tropics to avoid the singularity at the critical latitude for Rossby wave propagation. Valdes and Hoskins (1989) used a high-resolution linear model to investigate its response to orography and diabatic heating derived from operational weather analysis. They suggested that tropical diabatic heating is the most important for extratropical stationary waves while orography explains $30 \%$ of the amplitude. On the other hand, Huang and Gambo (1982) emphasized extratropical thermal forcing. These simple models are very useful for understanding the formation of stationary eddies, but the quantitative use of these linear model results is subject to the sensitivity to the choice of the basic state, damping coefficients, and estimated diabatic heating.

AGCMs have also been used to assess relative importance of different forcings to create stationary eddies. Using a Geophysical Fluid Dynamics Laboratory-National Oceanic and Atmospheric Administration (NOAA) AGCM, Manabe and Terpstra (1974) compared experiments with and without mountains. Based on their results, Held (1983) suggested that orographically forced waves have about the same amplitude as thermally forced ones and that the former waves show stronger vertical propagation than the latter. With a Meteorological Research Institute of Japan's AGCM, Tokioka and Noda (1986) extended the above work by dividing the orographic forcing into that associated with the Tibetan Plateau and the Rockies-Greenland. Blackmon et al. (1987) examined tropical SST effects using a National Center for Atmospheric Research's (NCAR) Community Climate Model (CCM).

All the model studies pointed out the importance of thermal forcing for stationary eddies in the upper troposphere. In AGCMs, thermal forcing specified at the lower surface may further be divided into that due to land-sea distribution and to zonal variations of SST. Inatsu et al. (2000, hereafter IMX) conducted a set of experiments with the Center for Climate System Research (CCSR)/National Institute for Environmental Studies (NIES) AGCM under both idealized and realistic conditions, and found that the extratropical land-sea distribution is much less effective in forming the westerly jet core than the zonal variation in tropical SSTs. In this study, we will extend IMX's work by examining the three-dimensional structure of stationary eddies in response to three types of forcing: extratropical land-sea distribution, tropical SST variation, and orography. We will further investigate the relationship and interaction between stationary and transient eddies. The latter concerns the formation of the storm track. For this purpose, we have carried out perpetual January integrations for 1500 days, a period long enough to ensure stable statistics, which enable us to study the robustness of model stationary eddies. Motivated by the following previous studies, we will carry out detailed analysis of the model response to zonal variations in tropical SST.

SST-induced changes in tropical deep convection are known to affect extratropical atmosphere through socalled teleconnection (Horel and Wallace 1981). Geisler et al. (1985) showed that a geographically fixed PacificNorth America (PNA) pattern is excited by SST anomalies at various longitudes across the tropical Pacific in AGCM experiments. They also noted nonlinearity in the model response. While the tropical response increases at a faster than linear rate with tropical SST anomalies, the midlatitude response saturates when the imposed SST anomalies exceed a certain magnitude. By analyzing the response of a nonlinear model to several types of prescribed diabatic heating in the Tropics, Hendon (1986) showed that the linear-viscous approximation (e.g., Matsuno 1966; Gill 1980) is valid only for a weak tropical forcing. With a strong forcing, the subtropical anticyclone in the upper troposphere shifts eastward due to nonlinearity. However, Ting and Held (1990) noted insignificant nonlinearity in the response of their AGCM to strong zonal variations in tropical SSTs. We will examine this nonlinearity in extratropical stationary eddies.

Subtropical jet core and storm tracks in the winter extratropical climate interact intimately with each other. Blackmon et al. (1977) suggested that transient eddies induce an ageostrophic southerly wind, which decelerates the westerly jet speed. The northward transient heat flux weakens the baroclinic westerlies, while the transient momentum flux converges on the poleward edge of the jet exit, destroying the baroclinic jet structure. The high baroclinicity (Lindzen and Farrell 1980) off the east coast of large continents is hypothesized to be essential for the downstream development of storm tracks (Hoskins and Valdes 1990). Hoskins et al. (1983) suggested that while low-frequency eddies reduce the westerly wind speed at the jet exit, synoptic eddies accelerate barotropic westerlies in the storm track region by reducing the vertical wind shear at the entrance of the storm track. We will investigate the action of transient eddies on the time-mean flow in this study.

The rest of this paper is organized as follows. We describe the model and experimental design in section 2 , and extratropical and subtropical responses in section 3. Section 4 studies the formation mechanism of stationary waves forced by extratropical land-sea heat contrast and orography. Section 5 focuses on the tropical SST forcing mechanism. Section 6 is a summary.

\section{Model and experiments}

\section{a. Model}

Here, we use CCSR/NIES AGCM that is jointly developed by CCSR at University of Tokyo and NIES in 
( a ) Z ( OBS) @250hPa

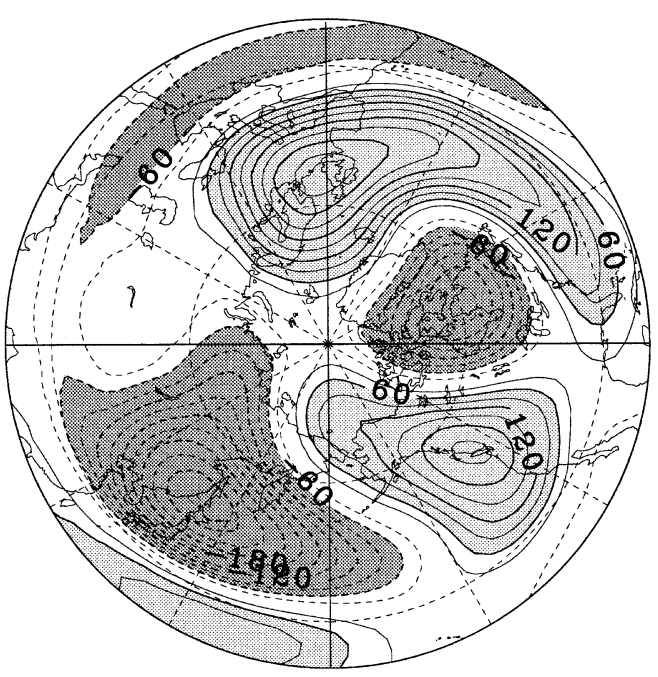

(c) $\operatorname{RMS}\left(\mathrm{Z}_{\mathrm{bp}}\right)(\mathrm{OBS}) @$ $250 \mathrm{hPa}$

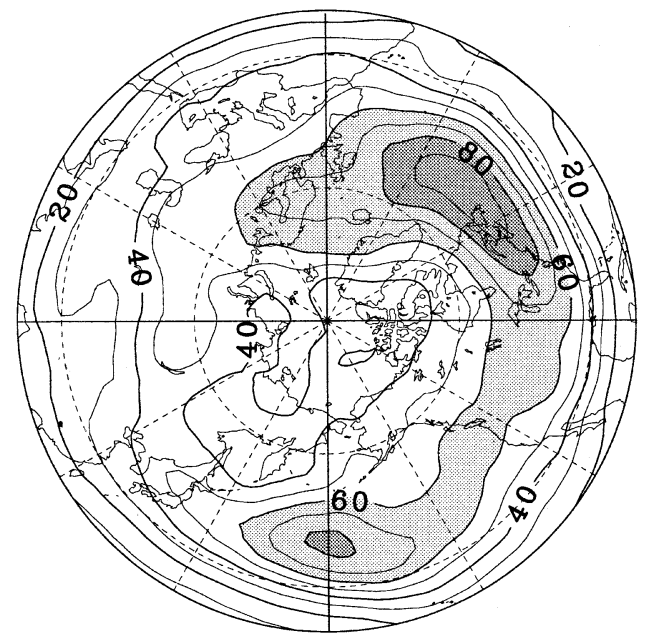

( b ) Z ( CTR)@250hPa

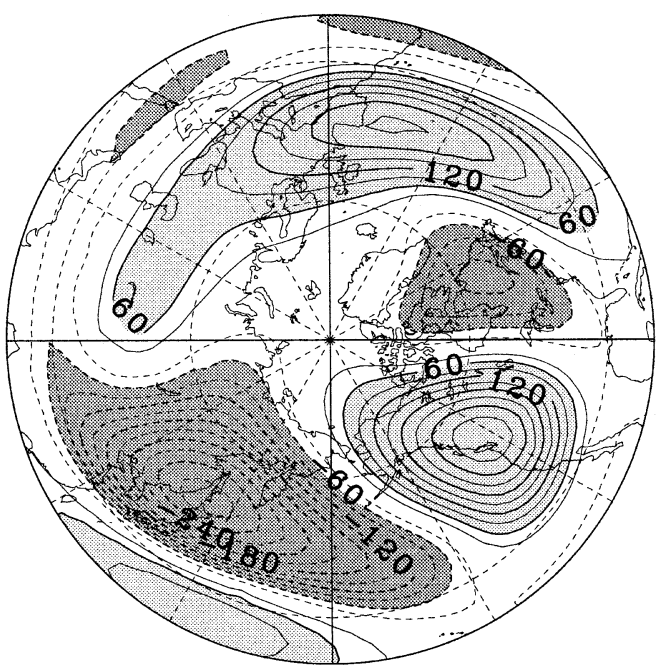

(d) $\operatorname{RMS}\left(\mathrm{Z}_{\mathrm{bp}}\right)(\mathrm{CTR})$ @2 $50 \mathrm{hPa}$

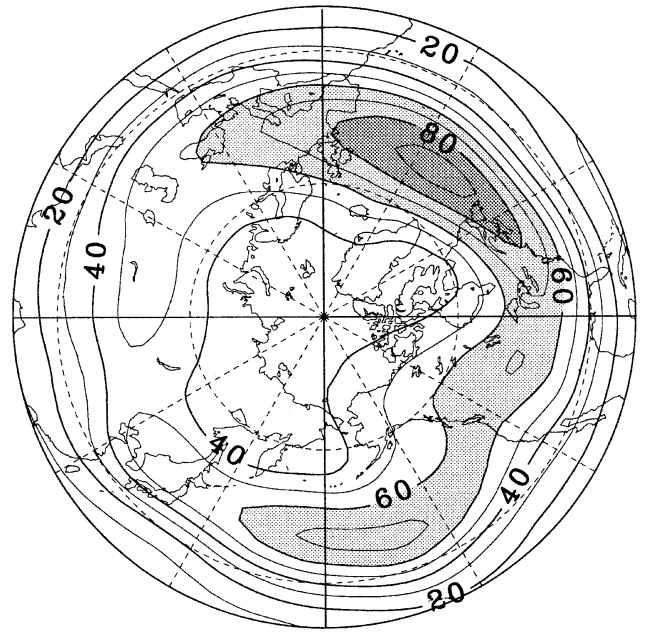

FIG. 1. Stationary eddy components of 250-hPa geopotential height in Jan averaged from 1990 to 1997 based upon European Centre for Medium-Range Weather Forecasts (ECMWF) data (a) and for CTR (b). Contour intervals are $30 \mathrm{~m}$ with negative contours dashed and zero contours removed. The light and heavy shadings are $<-60 \mathrm{~m}$ and $>60 \mathrm{~m}$, respectively. (c) (d) Same as the upper panels, but for synoptic eddy activity defined as std dev of 2.5-6-day geopotential height fluctuations. Contour interval $10 \mathrm{~m}$. The light and heavy shadings $>60 \mathrm{~m}$ and $>80$ $\mathrm{m}$, respectively.

Japan. This model is based upon the primitive equation on the sphere. The physics includes the parameterization for radiation, cumulus convection, large-scale condensation with prognostic cloud water, vertical diffusion, gravity wave drag, surface flux, and ground hydrology. See Numaguti et al. (1997) for a more detailed description of this model. This AGCM has been applied to studies of atmospheric hydrological cycle (Numaguti 1999 ) and interannual variability of the monsoon (Shen et al. 1998), and coupled with ocean models for climate studies (Watanabe et al. 1999; Xie and Saito 2001).

We use a version with a triangular truncation at the zonal wavenumber 42 in horizontal and 20 sigma levels in vertical. All numerical integrations are carried out under the perpetual January condition. To focus on the effect of zonal variations of SST, land-sea contrast, and orography, we set other surface boundary conditions zonally uniform: roughness, wetness, albedo, ozone, and 
TABLE 1. Experiment conditions.

\begin{tabular}{lllc}
\hline \hline Expt & Continent & \multicolumn{1}{c}{ SST } & Mountain \\
\hline A0 & Aqua Planet & $\alpha=0.0$ in Eq. (1) & No \\
A1 & Aqua Planet & $\alpha=1.0$ in Eq. (1) & No \\
A2 & Aqua Planet & $\alpha=2.0$ in Eq. (1) & No \\
A3 & Aqua Planet & $\alpha=3.0$ in Eq. (1) & No \\
A4 & Aqua Planet & $\alpha=4.0$ in Eq. (1) & No \\
A5 & Aqua Planet & $\alpha=5.0$ in Eq. (1) & No \\
A6 & Aqua Planet & $\alpha=6.0$ in Eq. (1) & No \\
A7 & Aqua Planet & $\alpha=7.0$ in Eq. (1) & No \\
A8 & Aqua Planet & $\alpha=8.0$ in Eq. (1) & No \\
I0 & Idealized & $\alpha=0.0$ in Eq. (1) & No \\
H0 & Idealized & $\alpha=0.0$ in Eq. (1) & $h=4000$ in Eq. (2) \\
CTR & Realistic & Realistic & Realistic \\
NM & Realistic & Realistic & No \\
Tx0 & Realistic & Zonally uniform & No \\
\hline
\end{tabular}

sea-ice thickness. After an 1-yr spinup period, the model outputs are stored twice daily for 1500 model days, including horizontal $[\mathbf{u}=(u, v)]$ and vertical velocity $(\omega)$, temperature $(T)$, geopotential height $(z)$, streamfunction, vorticity $(\zeta)$, velocity potential, and divergence $(D)$ on the isobar surfaces of 1000, 950, 900, 850, 700, $500,400,300,250,200,150,100,70,50,30,20$, and $10 \mathrm{hPa}$, rainfall, SLP, and surface air temperature (SAT). All the statistics presented are based on the 1500-day average.

\section{b. AGCM performance}

We conduct a control simulation (CTR) with the observed climatological SST and orography and compare it with observations. Figures $1 \mathrm{a}$ and $1 \mathrm{~b}$ compare 250 $\mathrm{hPa}$ geopotential height between the observed January climatology and the 1500-day model average. The extratropical stationary waves in CTR are in a reasonable agreement with observations. The anticyclone over the northeastern Pacific is slightly strong while the amplitude of the cyclone over Hudson Bay and the anticyclone over Europe are slightly weak compared with the observed climatology.

We next compare synoptic transient eddies. Following Blackmon (1976), the transient eddy activity is defined as the standard deviation of geopotential height variation with 2.5-6-day periods. The regions of high eddy activity in the upper troposphere, that is, the storm tracks, are found over the Pacific and the Atlantic in both observation and CTR (Figs. 1c and 1d). The position and strength of these storm tracks in CTR closely resemble those in observations, except that the Atlantic storm track extends too south into Europe in the model. This may be related to weaker diffluence of the time-mean flow over the Atlantic (Fig. 1b).

\section{c. Experiment designs}

We conduct a set of idealized experiments designed to isolate the effect of surface boundary forcing (Table 1). Letter " $A$ " stands for Aqua Planet experiments, with SSTs given by

$$
T_{*}(\lambda, \phi)= \begin{cases}T_{0}+T_{2} \sin ^{2}\left(\phi-\phi_{0}\right)+\frac{\alpha}{2} \sin \lambda \exp \left(-\frac{\phi^{2}}{200}\right) & \text { for } T_{*}>271.4 \\ 271.4 & \text { elsewhere }\end{cases}
$$

where $\lambda$ and $\phi$ denote longitude and latitude, respectively. Here $T_{0}=301.15 \mathrm{~K}, T_{2}=-32.0 \mathrm{~K}, \phi_{0}=8^{\circ} \mathrm{S}$, and $\alpha=0,1, \ldots$, and 8 for $\mathrm{A} 0, \mathrm{~A} 1, \ldots$, and $\mathrm{A} 8$, respectively. For example, the prescribed SST distribution in A5 is displayed in Fig. 2. An idealized "Eurasian" continent that spans from $90^{\circ} \mathrm{E}$ to $90^{\circ} \mathrm{W}$, north of $20^{\circ} \mathrm{N}$, is placed in experiments labeled $\mathrm{I} 0$ and $\mathrm{H} 0$, with zonally uniform SSTs. Land surface is flat at sea level in I0, while a large-scale mountain with a height distribution,

$$
\begin{aligned}
& H_{*}(\lambda, \phi) \\
& \quad=h \exp \left[-\left(\frac{\phi-35^{\circ}}{10}\right)^{2}\right] \exp \left[-\left(\frac{\lambda-180^{\circ}}{60}\right)^{2}\right],
\end{aligned}
$$

with $h=4000 \mathrm{~m}$, is used in H0. The designs of the $\mathrm{A} 0, \mathrm{~A} 1, \ldots, \mathrm{A} 8, \mathrm{I} 0$, and $\mathrm{H} 0$ runs allow us to isolate and study the effects of tropical SST, land-sea distribution and orographic forcings.

\section{Extratropical and subtropical response}

In this section, we describe the results from four experiments, $\mathrm{A} 0, \mathrm{~A} 5, \mathrm{I} 0$, and $\mathrm{H} 0$ (Table 1). By comparing these runs, we will investigate the response to extratropical land-sea contrast, large-scale mountain, and tropical SST variation. More detailed analyses will follow in sections 4 and 5 .

\section{a. Zonal-mean structure}

Figure 3 displays zonal-mean zonal wind velocity and meridional circulation for A0, A5 minus A0, I0 minus $\mathrm{A} 0$, and $\mathrm{H} 0$ minus $\mathrm{A} 0$. Figures $3 \mathrm{~b}-\mathrm{d}$ illustrate the effects 


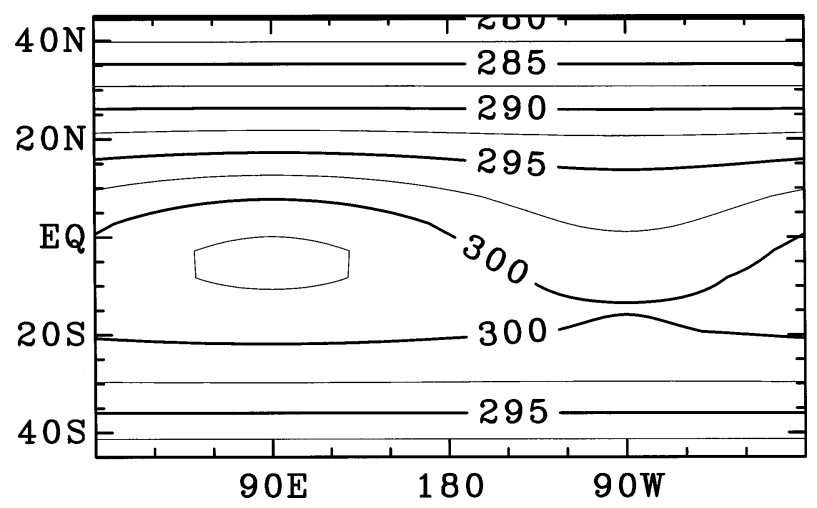

FIG. 2. The prescribed SST distribution for experiment A5. Contour interval is $2.5 \mathrm{~K}$.

of tropical SST variation, extratropical land-sea contrast, and large-scale mountain plus land-sea distribution. The subtropical westerly jet is centered at $30^{\circ} \mathrm{N}$ and $250 \mathrm{hPa}$. Its maximum speed is about $60 \mathrm{~m} \mathrm{~s}^{-1}$, but is slightly weaker at $50 \mathrm{~m} \mathrm{~s}^{-1}$ in A5. Consistent with the theory of the axisymmetric flow (Held and Hou 1980), the subtropical jet is located in the descending branch of the Hadley cell. In I0, the upper branch of Hadley circulation extends slightly poleward so that the westerly jet axis shifts northward (Fig. 3c). In I0 and $\mathrm{H} 0$, strong cooling occurs in the extratropical lower atmosphere (light shading in Figs. 3c and 3d) due to radiative cooling over the continent. Such an increased temperature gradient leads to an increased vertical shear of westerly wind in the extratropics. High-latitude temperature increases as indicated by the heavy shade above the tropopause in $\mathrm{I} 0$ and $\mathrm{H} 0$ are indicative of the poleward heat flux associated with vertically propagating Rossby waves (Andrews et al. 1987).

In the Tropics, easterly winds prevail in the upper troposphere for A0, I0, and $\mathrm{H} 0$, but westerly for A5, where the subtropical jet is much broader and extends into the equator. The equatorial superrotation in A5, unrealistic in some sense, takes place. This corresponds to westerly duct over the eastern equatorial Pacific during a strong La Niña winter (cf. Matthews and Kiladis 1999). The A5 minus A0 difference is consistent with the westerly deceleration (acceleration) due to the momentum flux divergence (convergence) associated with the generation (dissipation) of Rossby wave (Edmon et al. 1980) in the extratropics (Tropics). See Hoskins et al. (1999) for a more detailed discussion. Thus, the modulation of the Rossby waves forced by zonal variation in surface boundary conditions causes changes in the zonal-mean circulation of the atmosphere. The rest of this paper will focus on the generation and propagation of the stationary waves.

\section{b. Stationary eddies}

Figure 4 displays the stationary eddy geopotential height and zonal wind in the upper troposphere. In

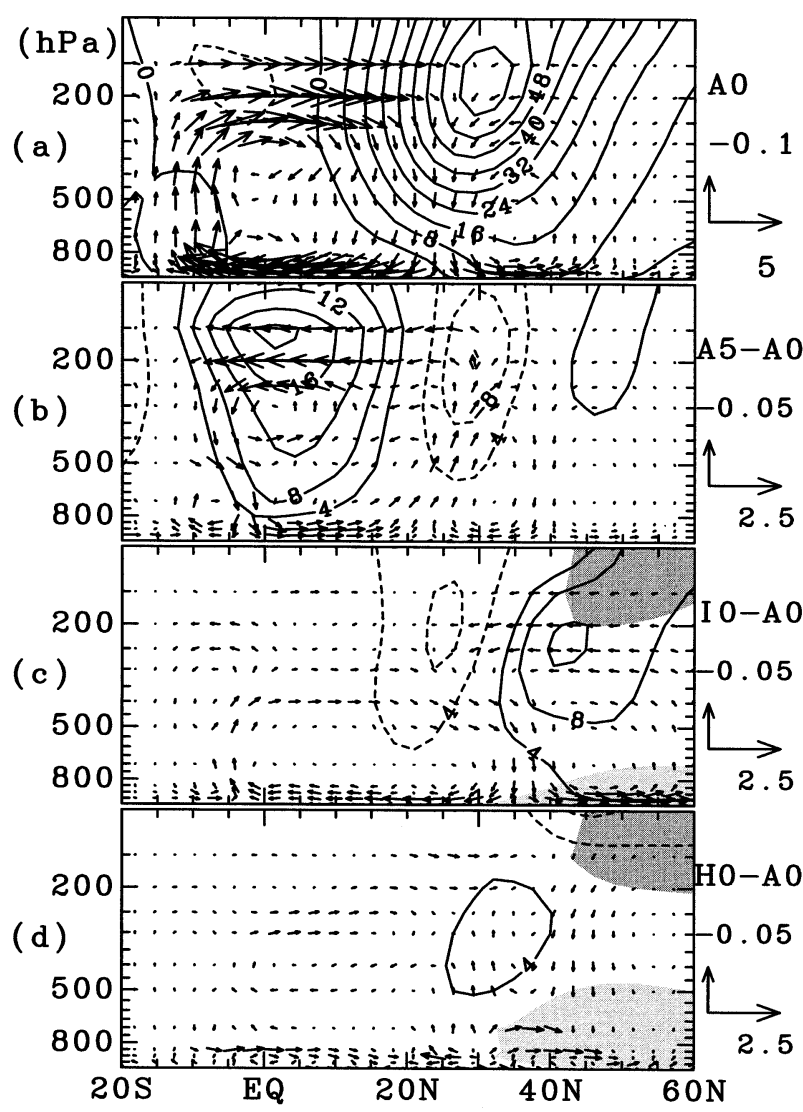

FIG. 3. (a) Zonal-mean and time-mean zonal wind velocity (contours) and meridional circulation (vectors) for A0. Contour intervals are $8 \mathrm{~m} \mathrm{~s}^{-1}$ with negative contours dashed. The reference arrows on the right are $5 \mathrm{~m} \mathrm{~s}^{-1}$ and $-0.1 \mathrm{hPa} \mathrm{s}^{-1}$ for the meridional and vertical velocities, respectively. Differences from the A0 run of the zonal velocity (contours), meridional circulation (vectors), and temperature (shading) in (b) A5, (c) I0, and (d) H0. Contour intervals are $4 \mathrm{~m} \mathrm{~s}^{-1}$ with negative contours dashed and zero contours removed. The reference vectors are $2.5 \mathrm{~m} \mathrm{~s}^{-1}$ and $-0.05 \mathrm{hPa} \mathrm{s}^{-1}$. The light and heavy shadings are $<-5 \mathrm{~K}$ and $>5 \mathrm{~K}$, respectively.

I0, the subtropical jet speed shows small zonal variations with a range less than $5 \mathrm{~m} \mathrm{~s}^{-1}$ (Fig. 4d). Modest geopotential height variations of zonal wavenumber one are found around $60^{\circ} \mathrm{N}$ (Fig. 4a). In H0, centers of eddy geopotential heights are located in lower latitudes (Fig. 4b), and the zonal variation in subtropical westerly speed is much stronger than in I0. As the isolated orographic forcing contains many zonal wavenumber components, smaller-scale response is also evident in $\mathrm{H} 0$.

Whereas stationary eddies are largely confined in mid- and high latitudes in $\mathrm{H} 0$, large eddy geopotential heights are found in both the extratropics and the subtropics in A5. With sign change across $30^{\circ} \mathrm{N}$, these eddies induce large variations in the westerly jet speed. The speed maximum (minimum) of the subtropical westerlies is located roughly to the north of the SST maximum (minimum) in the Tropics. 


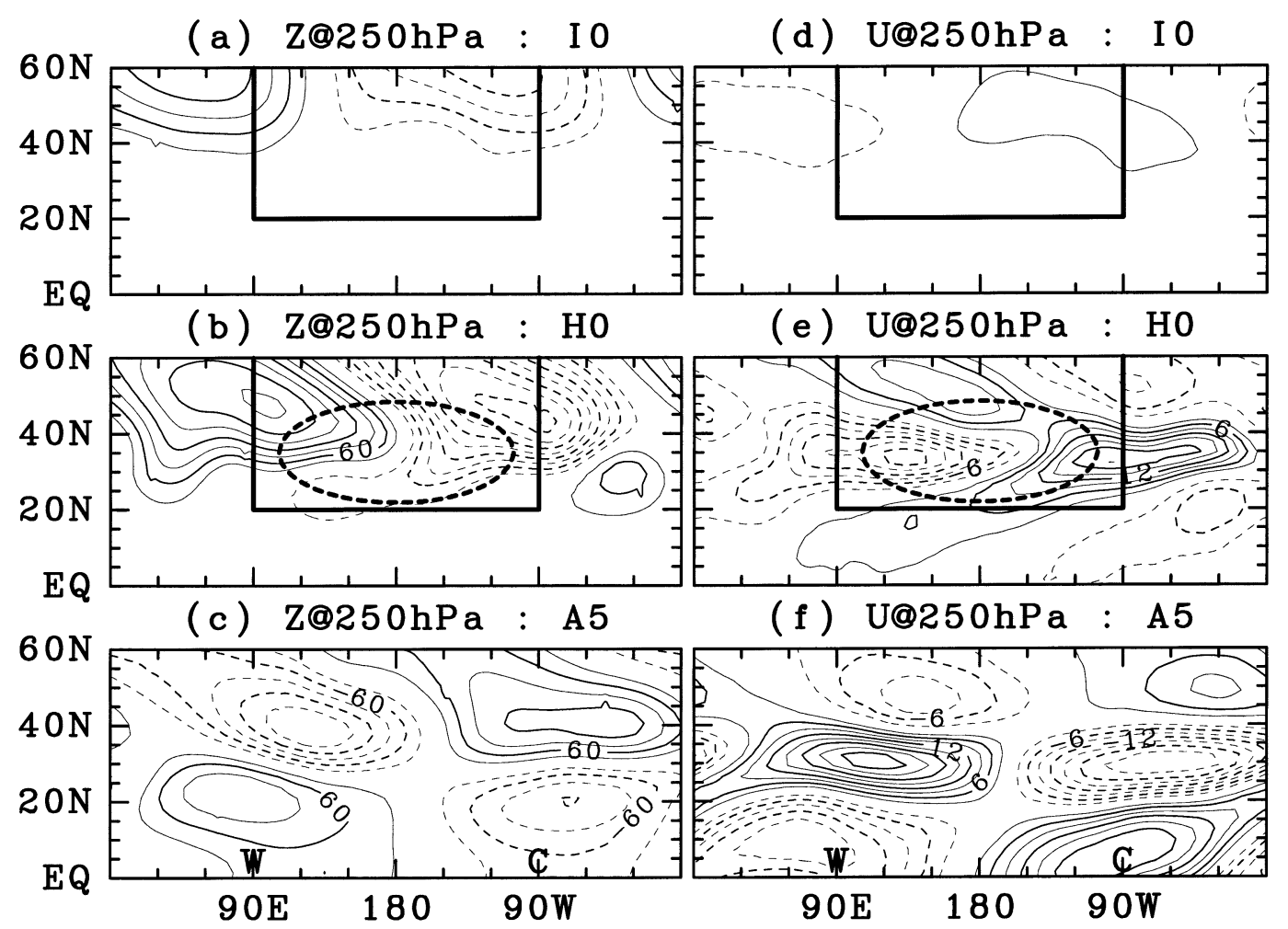

FIG. 4. (a)-(c) Stationary eddy components of geopotential height and (d)-(f) zonal wind on $250 \mathrm{hPa}$ for (a), (d) I0, (b), (c) H0, and (c), (f) A5. Contour intervals are (a)-(c) $30 \mathrm{~m}$ and (d)-(f) $3 \mathrm{~m} \mathrm{~s}^{-1}$ with negative contours dashed and zero contours removed. The thick solid lines denote the coastline of the idealized continent. The thick dotted line is $800 \mathrm{~m}$ above the sea level. The $\mathrm{W}$ and $\mathrm{C}$ in (c) and (f) are positioned at the center of the prescribed tropical warm and cold water pools, respectively.

Large zonal asymmetry appears not only in the upper troposphere but also in SLP and SAT (Fig. 5). In I0 and HO, cold air occupies the continent while SAT is largely determined by prescribed SST over the ocean because of strong heat exchange across the air-sea interface. Such zonal SAT variation induces high (low) SLP over the land (ocean), corresponding to the observed Eurasian high (Aleutian low). See the next section for a detail diagnostic analysis for both $\mathrm{I} 0$ and HO. Whereas little zonal asymmetry appears in the Tropics for these two experiments, a pair of low and high SLP centers appear in the Tropics in A5, over the warm and cold SST, respectively. North of $30^{\circ} \mathrm{N}$ in A5, SLP is in phase with the upper tropospheric geopotential height, indicative of barotropic Rossby waves (Fig. 7c). In the subtropics, by contrast, the first baroclinic mode of Rossby waves dominates in eddy geopotential height fields (Fig. 7d).

Plumb (1985) proposed an useful diagnostic quantity to trace the three-dimensional propagation of stationary Rossby waves, which is called stationary wave activity flux $\mathbf{F}_{s}$, defined as

$$
\mathbf{F}_{s}=p \cos \phi\left(\begin{array}{c}
\bar{v}^{*} \bar{v}^{*}-\frac{g}{2 f} \frac{\partial}{\partial x}\left(\bar{v}^{*} \bar{z}^{*}\right) \\
-\bar{u}^{*} \bar{v}^{*}-\frac{g}{2 f} \frac{\partial}{\partial x}\left(\bar{u}^{*} \bar{z}^{*}\right) \\
\frac{f}{S}\left[\bar{v}^{*} \bar{T}^{*}-\frac{g}{2 f} \frac{\partial}{\partial x}\left(\bar{T}^{*} \bar{z}^{*}\right)\right]
\end{array}\right),
$$

where the overbar denotes the time average, the asterisk the zonal deviation, $p$ the sigma pressure, $g$ the gravity acceleration rate, $f$ the Coriolis parameter, and $S$ static stability averaged over the Northern Hemisphere. The zonal averages of $\mathbf{F}_{s}$ is equal to the Eliassen-Palm flux (Edmon et al. 1980). In the limit of plane waves, $\mathbf{F}_{s}$ is parallel to the group velocity of Rossby wave.

Figure 6 displays 250-hPa horizontal (vectors) and 500-hPa vertical (contours) wave activity flux for I0 and $\mathrm{H} 0$. While wave activity flux is insignificant everywhere in $\mathrm{I} 0$, wave propagation is pronounced in $\mathrm{H} 0$ : vertical wave activity flux is quite large around $40^{\circ} \mathrm{N}$ corresponding to the northern edge of the prescribed mountain; the horizontal component of wave activity flux is 
(a) SLP TS : I0

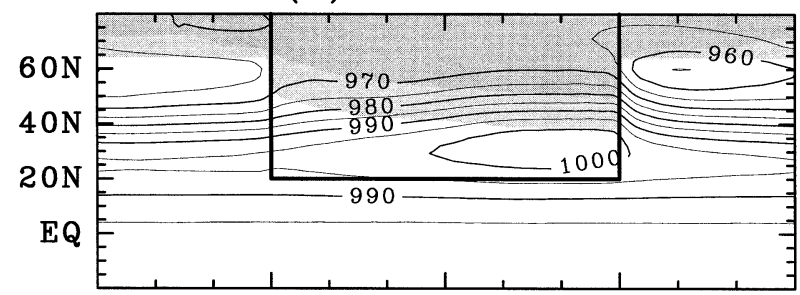

(b) SLP Ts : HO

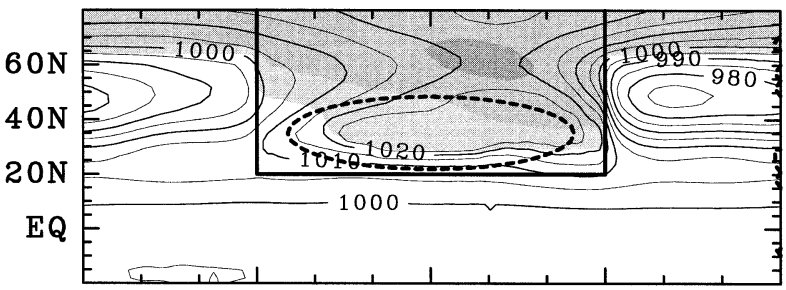

(c) SLP Ts : $\mathrm{A5}$

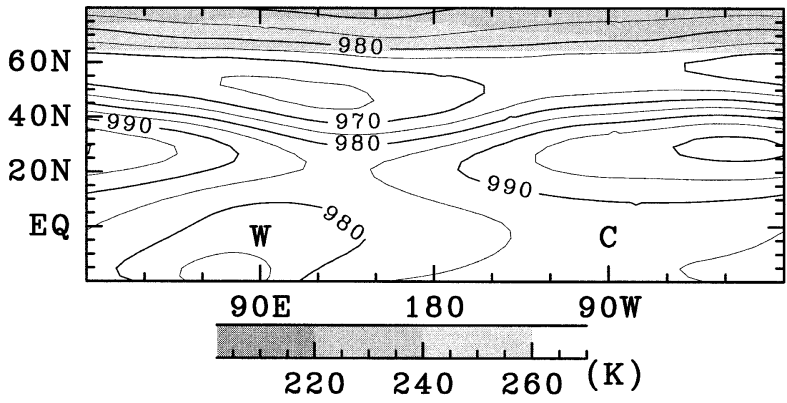

FIG. 5. SLP (contours) and SAT (shading) for (a) I0, (b) HO, and (c) A5. Contour intervals are $5 \mathrm{hPa}$. The temperature $(\mathrm{K})$ shadings are as the grayscale on the bottom for reference. The $\mathrm{W}$ and $\mathrm{C}$ notations are as in Fig. 4.

strongly divergent along $35^{\circ} \mathrm{N}$, the latitude of the maximum elevation, indicative of a tendency for the waves to propagate away from the mountain.

The vectors in Fig. 7 show the wave activity flux $\mathbf{F}_{s}$. Stationary eddies induced by extratropical and tropical forcings are quite different in their vertical propagation. Extratropical land-sea contrast forces little amplitude of Rossby waves and their wave flux does not propagate into the stratosphere in the midlatitudes (Fig. 7a). The large-scale mountain is very effective in causing vertical propagation of stationary waves (Fig. 7b). The response to the tropical forcing, by contrast, shows little vertical propagation (Fig. 7c).

\section{c. Internal variability}

The extratropical atmosphere contains high internal variability, which can mask stationary eddies. Here we assess how robust those stationary eddies are in our 1500-day integrations. We apply the Fourier expansion to a model variable $q(\lambda, t)$,

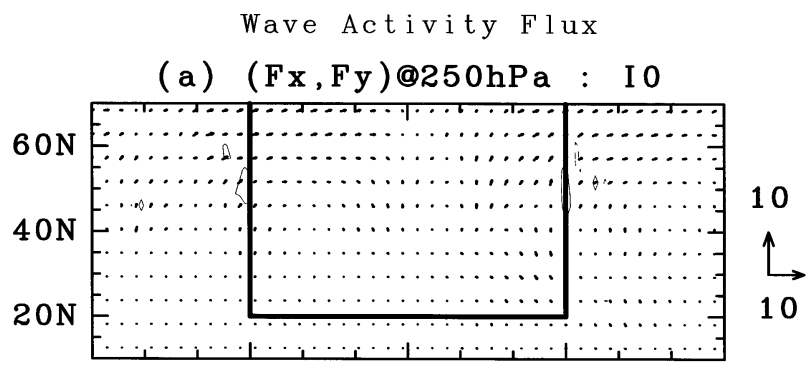

(b) $(\mathrm{Fx}, \mathrm{Fy}) @ 250 \mathrm{hPa}: \mathrm{HO}$

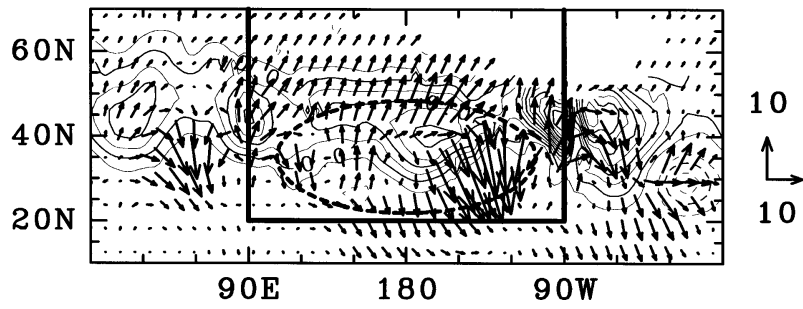

FIG. 6. Horizontal components of wave activity flux at $250 \mathrm{hPa}$ (vectors) and vertical component at $500 \mathrm{hPa}$ (contour) for (a) I0 and (b) H0. Contour intervals are $0.05 \mathrm{~m}^{2} \mathrm{~s}^{-2}$. The reference arrows on the right denote $x$ and $y$ components of $10 \mathrm{~m}^{2} \mathrm{~s}^{-2}$ and $10 \mathrm{~m}^{2} \mathrm{~s}^{-2}$, respectively. Arrows are plotted only in regions with westerly winds.

$$
q(\lambda, t)=a_{0}(t)+\sum_{n=1}^{\infty}\left[a_{n}(t) \cos n \lambda+b_{n}(t) \sin n \lambda\right],
$$

where $a_{0}$ is the zonal mean, and $a_{n}$ and $b_{n}$ are the Fourier expansion coefficients that represent the amplitudes of stationary eddies. Here we focus on the wavenumberone component, which dominates the model response. Figure 8 is a schematic example of a $\left[a_{1}(t), b_{1}(t)\right]$ scatterplot. The amplitude and phase of the wavenumberone component of the stationary eddies are defined as $R=\sqrt{\bar{a}_{1}^{2}+\bar{b}_{1}^{2}}$ and $\varphi=\arg \left(\overline{a_{1}}+\overline{b_{1}} \sqrt{-1}\right)$, respectively. The standard deviation around the centroid of the scatter in $a_{1}-b_{1}$ phase space, $S=\sqrt{\overline{\left(a_{1}^{\prime 2}+b_{1}^{\prime 2}\right)}}$, will tell us the stationarity of the wavenumber-one component. Here the prime denotes the deviation from the time average. The stationary response with $R>S$ can be regarded as robust. Otherwise, the wavenumber-one wave changes its phase and amplitude so much that stationary component is hardly recognizable in a snapshot or monthly mean map.

Figures $9 \mathrm{a}-\mathrm{c}$ display the scatterplots of selected variables in I0. The SAT pattern (Fig. 9a) is highly stationary, with very low values below the freezing point over the continent as south as $30^{\circ} \mathrm{N}$ in this perpetual January run. Over the model ocean, they are strongly tied to prescribed SSTs and remain warm. Despite this robust zonal variation in SATs, stationary eddies in the upper troposphere are weak, and the wavenumber-one amplitude does not exceed the noise level even in high latitudes (Figs. 4a and 9c). The small amplitude, in combination with their high-latitude location, makes these stationary eddies ineffective in modulating the subtrop- 
(a) Z@46N: I 0

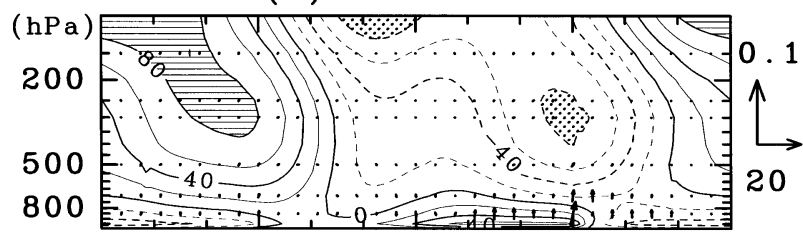

(b) $\mathrm{Z@46N} \mathrm{:} \mathrm{HO}$

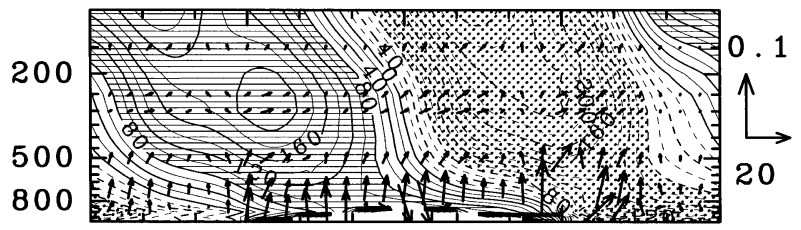

(c) $\mathrm{Z} @ 46 \mathrm{~N}: \mathrm{A} 5$

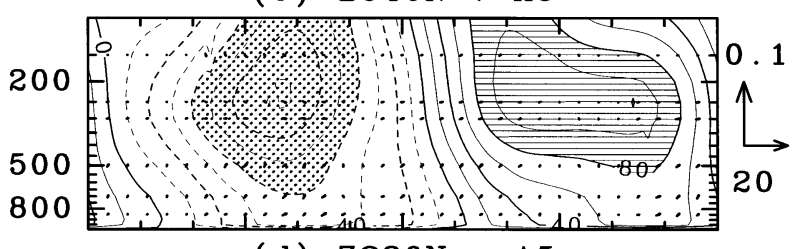

(d) Z@20N : A5

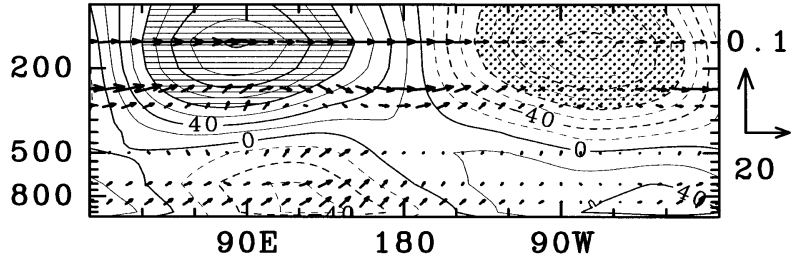

FIG. 7. Vertical cross sections of the stationary eddy geopotential heights (contours and shading) and wave activity flux (vectors) in (a) I0, (b) $\mathrm{H} 0$, (c) A5 at $46^{\circ} \mathrm{N}$ and (d) A5 at $20^{\circ} \mathrm{N}$. Contour interval is $20 \mathrm{~m}$ with negative contours dashed. Dotted shading $<-80 \mathrm{~m}$; stripe shading $>80 \mathrm{~m}$; the horizontal and vertical flux reference vectors are $20 \mathrm{~m}^{2} \mathrm{~s}^{-2}$ and $0.1 \mathrm{~m}^{2} \mathrm{~s}^{-2}$, respectively.

ical jet (Fig. 9b). Adding a large-scale mountain in H0 leads to a stronger and more robust stationary response in midlatitudes (not shown).

In A5, by contrast, the stationary eddies are very strong in the subtropics, and their time-mean amplitudes are more than three times the chaotic variability (Fig. 9d). The high-latitude response is also robust and twice of the standard deviation of internal noise (Fig. 9f). Thus, the wavenumber-one modulation of the subtropical jet stands out as highly robust in response to tropical SST forcing (Fig. 9e).

\section{d. Transient eddies}

The surface forcing described above modulates not only stationary but also transient eddies, due to their mutual interaction. As reviewed in the introduction, baroclinic eddies are generally considered to develop downstream of the westerly jet core in the extratropics (Blackmon et al. 1977; Hoskins and Valdes 1990).

Figures 10 and 11 display the statistics of the upper

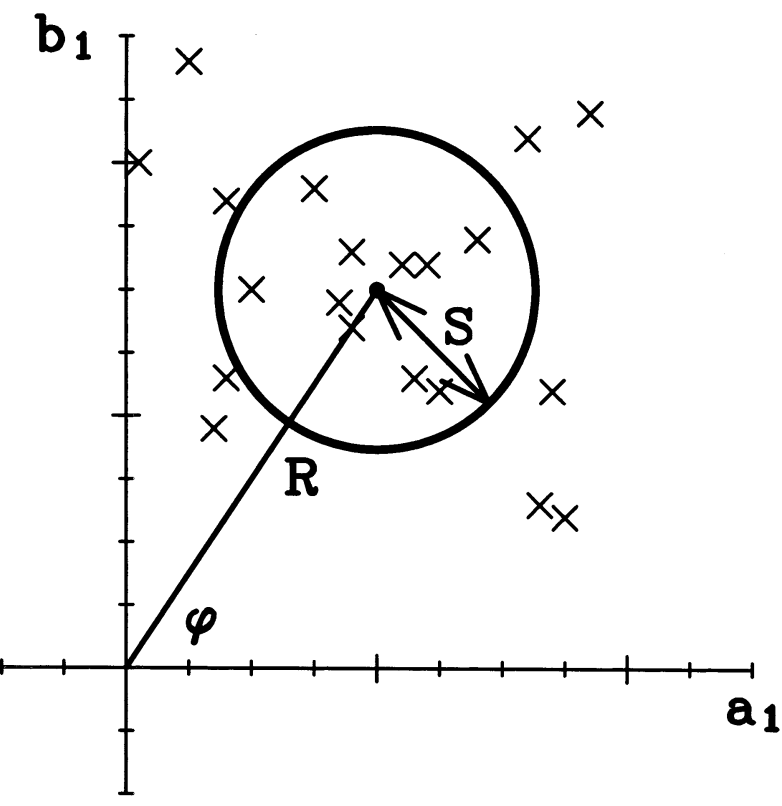

FIG. 8. Illustration for scatterplots of the Fourier expansion coefficients $\left[a_{1}(t), b_{1}(t)\right]$ in Eq. (4). Parameters $R$ and $\varphi$ are the amplitude and phase of a stationary wave. Parameter $S$ is the std dev of the scatter or the average distance in $a_{1}-b_{1}$ phase space from the time mean of $\left[a_{1}(t), b_{1}(t)\right]$

and lower tropospheric transient eddy activity, respectively. See section $2 b$ for the definition of transient eddy activity. In I0, the storm track at $250 \mathrm{hPa}$ is nearly uniform in the zonal direction (Fig. 10a), except for a weak maximum in the central continent. Rainfall (shading in Fig. 11a) is concentrated over the ocean in the midlatitudes. Such a strong zonal asymmetry in rainfall is associated with the availability of moisture rather than due to changes in storm activity. The eddy heat flux at $850 \mathrm{hPa}$ (contour in Fig. 11a) is large over the high-latitude ocean and the midlatitude continent, regions of large meridional temperature gradient (Fig. $5 a)$. In contrast with the nearly zonally uniform distribution of upper-level eddy activity in I0, storm track is localized over the ocean in HO (Fig. 10b), and so are the precipitation and eddy heat flux (Fig. 11b). In A5, storm track in the upper troposphere develops downstream of the jet core in association with enhanced heat flux near the surface. As in observations, the upper-level storm activity extends farther downstream compared with the heat flux, which is indicative of the baroclinic growth of the transient eddies. The storm track is lightly tilted northeastward like the Atlantic storm track and has a much broader meridional scale than the band of maximum eddy activity to the west. The latter is indicative of Rossby wave radiation into lower and higher latitudes (cf. Hoskins et al. 1983).

Given the enhanced baroclinicity off the east coast and high precipitation over the ocean in $\mathrm{I} 0$, it is some- 

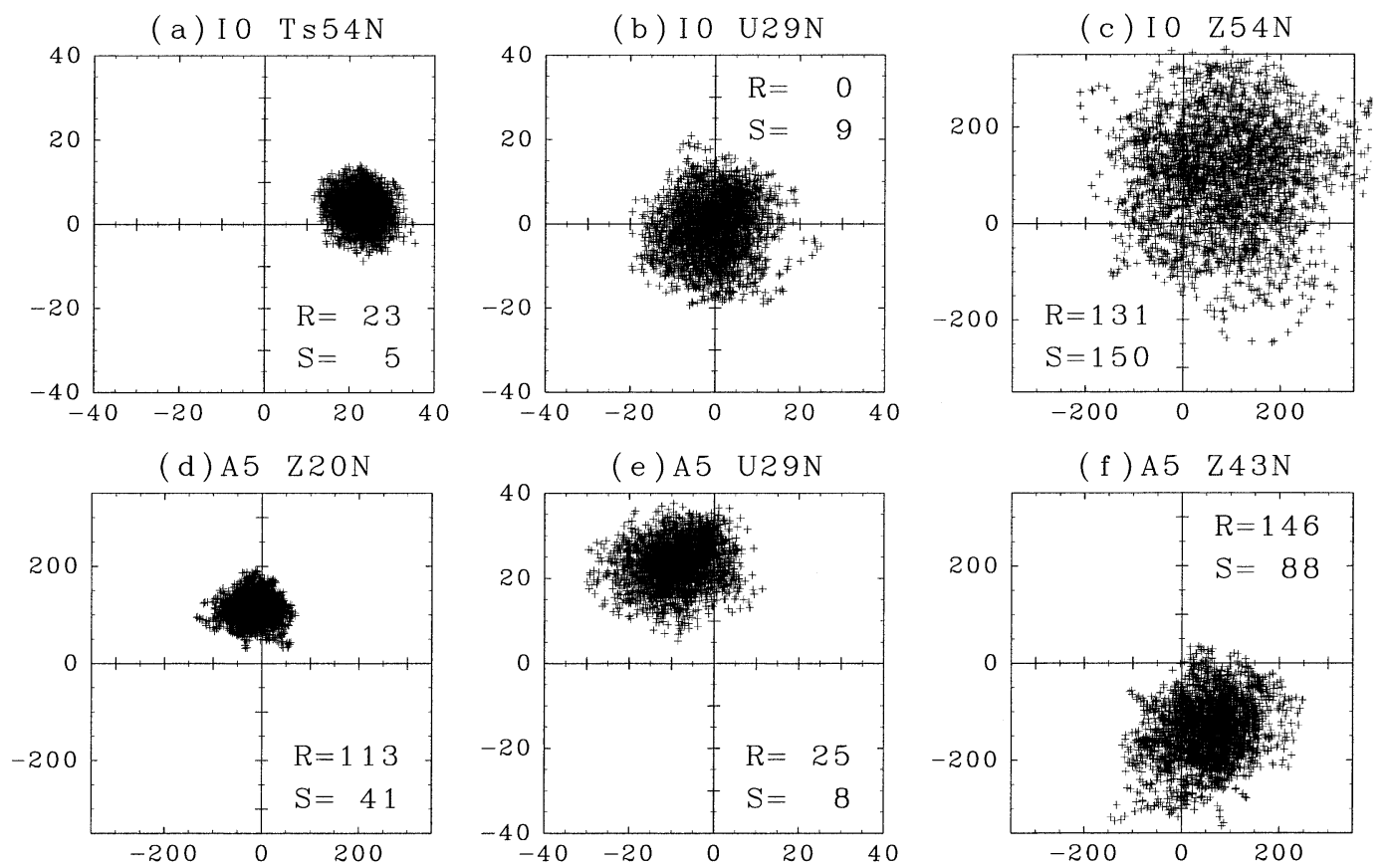

FIG. 9. Scatterplots of $\left[a_{1}(t), b_{1}(t)\right]$ for surface temperature at (a) $54^{\circ} \mathrm{N}$, (b) zonal wind at $29^{\circ} \mathrm{N}$ and $250 \mathrm{hPa}$, and (c) geopotential height at $54^{\circ} \mathrm{N}$ and $250 \mathrm{hPa}$ in I0. (d) Geopotential height at $20^{\circ} \mathrm{N}$ and $250 \mathrm{hPa}$, (e) zonal wind at $29^{\circ} \mathrm{N}$ and $250 \mathrm{hPa}$, and (f) geopotential height at $43^{\circ} \mathrm{N}$ and $250 \mathrm{hPa}$ in A5. See the text and Fig. 8 for details.

what surprising that the storm track is rather zonally uniform and the weak maximum in storm activity is located on the eastern continent than off the coast in the ocean, contrary to observations. Following this set of three experiments, the orographic and tropical SST forcings appear important in forming a localized storm track downstream of the westerly jet core. In the real atmosphere, they work cooperatively to produce the observed storm track, which is much more concentrated than in either $\mathrm{H} 0$ or $\mathrm{A} 5$.

\section{Extratropical forcing mechanism}

In this section, we analyze the budget of the timemean thermodynamical equation in order to reveal the mechanism to form stationary eddies by the extratropical forcings.

\section{a. Extratropical land-sea forcing}

The time-mean thermodynamical equation is written as,

$$
\begin{aligned}
& \bar{u} \frac{\partial \bar{\theta}}{\partial x}+\bar{v} \frac{\partial \bar{\theta}}{\partial y}+\bar{\omega} \frac{\partial \bar{\theta}}{\partial p}+\boldsymbol{\nabla} \cdot \overline{\mathbf{u}^{\prime} \theta^{\prime}}=\bar{Q},
\end{aligned}
$$

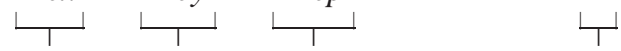

where $(x, y)=(a \lambda \cos \phi, a \phi), a$ is the earth's radius, $\theta$ potential temperature, and $Q$ is diabatic heating. The terms (a), (b), and (c) in Eq. (5) indicate time-mean zonal, meridional, and vertical advection of potential temperature. The diabatic heating in this analysis is obtained as the sum of all the terms in the left-hand side of in Eq. (5).

Figure 12 depicts longitude-height cross section of terms (a), (b), (c), and (d) in Eq. (5) at $40^{\circ} \mathrm{N}$ for I0. In the lower troposphere, the zonal advection term (a) has large positive values only at the eastern edge of the continent, while it is negative in the other region. The meridional advection term (b) has large negative values of about $-3 \mathrm{~K} \mathrm{day}^{-1}$ just above the continent, and it becomes approximately zero above the ocean. The term (c) is almost zero near the surface. Since the heat flux divergence associated with transient eddies has negligibly small values near the ground, consistent with the maximum of northward transient heat flux at $40^{\circ} \mathrm{N}$ (Fig. 11a), the diabatic heating term (d) is nearly balanced by the sum of terms (a), (b), and (c). Strong localized diabatic heating at the eastern edge of the continent balances the strong cooling due to the zonal advection. Over the rest of the continent, diabatic cooling dominated above the continent is balanced by northward meridional temperature advection (Fig. 12b; see also Fig. 5a). This explains the anticyclone (cyclone) near the surface in the eastern (western) coast of the continent, consistent with the Hoskins and Karoly (1981) scenario for response due to shallow heating in the midlatitudes.

On the other hand, it is much more difficult to explain the generation mechanism for the upper tropospheric 
(a) $\operatorname{RMS}\left(\mathrm{Z}_{\mathrm{bp}}\right) @ 250 \mathrm{hPa}: \mathrm{I}_{0}$

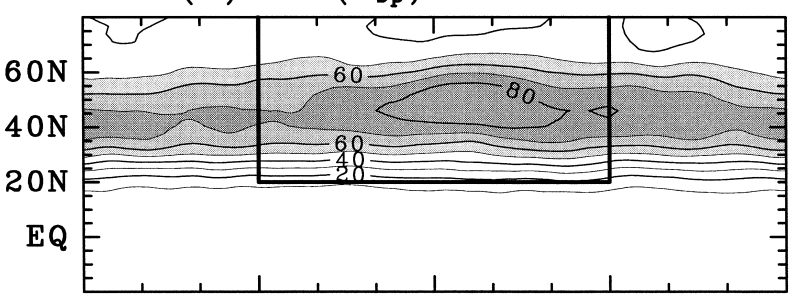

(b) $\operatorname{RMS}\left(\mathrm{Z}_{\mathrm{bp}}\right) @ 250 \mathrm{hPa}: \mathrm{HO}$

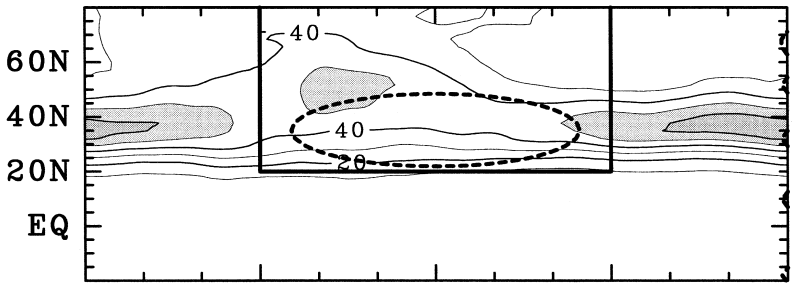

(c) $\operatorname{RMS}\left(\mathrm{Z}_{\mathrm{bp}}\right) @ 250 \mathrm{hPa}: \mathrm{A} 5$

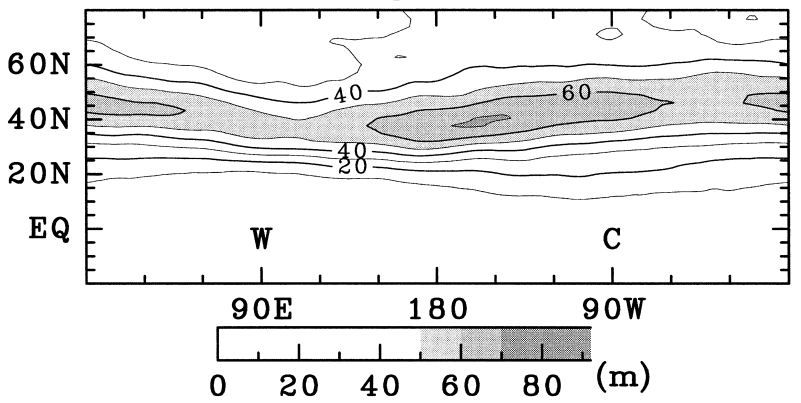

FIG. 10. Same as Fig. 5, but for transient eddy activity at $250 \mathrm{hPa}$. Contour intervals are $10 \mathrm{~m}$.

stationary eddies in the high latitudes in I0. Since the wave activity flux is very small except near the surface (Fig. 7a), upper tropospheric eddies could be independent of near-surface ones. As upper tropospheric stationary eddies are not robust for I0 compared with the chaotic noise level (Fig. 9c), we will not discuss further their formation mechanism.

\section{b. Extratropical orographic forcing}

We next examine the formation mechanism at stationary eddies in $\mathrm{H} 0$ by extratropical orographic forcing. For this analysis, we use the following time-mean vorticity equation:

$$
\begin{aligned}
& \bar{u}_{\psi} \frac{\partial \bar{\zeta}}{\partial x}+\bar{v}_{\psi} \frac{\partial \bar{\zeta}}{\partial y}+\beta \bar{v}_{\psi}+(f+\bar{\zeta}) \bar{D} \\
& \vdash_{\text {(a) }}^{\downarrow_{\text {(b) }}^{\mid}} \underset{\text { (c) }}{\downarrow_{\text {(c) }}} \longmapsto_{\text {(d) }}^{\mid} \\
& +\underbrace{\overline{\mathbf{u}}_{x} \cdot \boldsymbol{\nabla}(f+\bar{\zeta})}+\underbrace{\boldsymbol{\nabla} \cdot \overline{\left(\zeta^{\prime} \mathbf{u}^{\prime}\right)}} \approx 0 .
\end{aligned}
$$

Here, $\beta=(1 / a)(d f / d \phi)$, and subscripts $\psi$ and $\chi$ indicate the rotational and divergent components of wind ve- (a) $\left(\mathrm{V}^{\prime} \mathrm{T}^{\prime}\right) @ 850 \mathrm{hPa}$ RAIN : I0

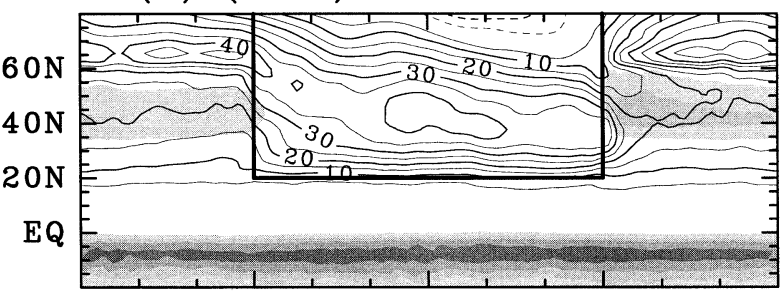

(b) ( $\left.\mathrm{V}^{\prime} \mathrm{T}^{\prime}\right) @ 850 \mathrm{hPa}$ RAIN : H0

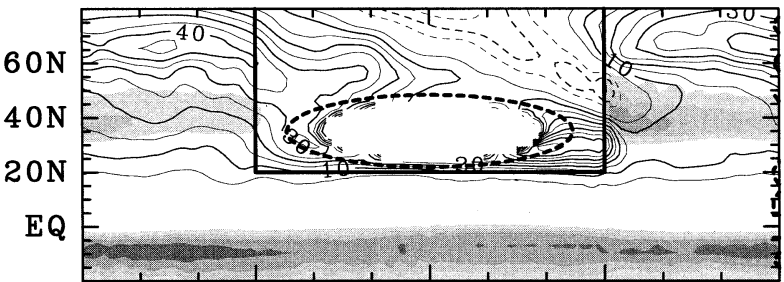

(c) $\left(\mathrm{V}^{\prime} \mathrm{T}^{\prime}\right) @ 850 \mathrm{hPa}$ RAIN : A5

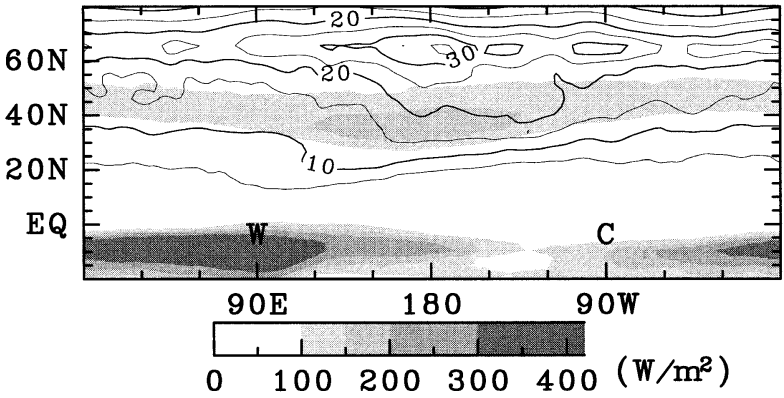

FIG. 11. Same as Fig. 5, but for transient eddy heat flux at 850 $\mathrm{hPa}$ (contours) and precipitation (shading). Contour intervals are 5 $\mathrm{K} \mathrm{m} \mathrm{s}^{-1}$ with negative contours dashed. Precipitation is in units of $\mathrm{W} \mathrm{m}^{-2}$, which represents heating in condensing water vapor. It is approximately equal to $1 \mathrm{~mm}$ month $^{-1}$.

locity, respectively. The terms (a) and (b) in Eq. (6) are the zonal and meridional advection of the time-mean relative vorticity by the rotational wind, respectively. The term (c) means the advection of the planetary vorticity by the rotational wind. The term (d) is the vortextube stretching effect. The term (e) means the horizontal advection of the planetary vorticity by divergent winds. The term (f) is the eddy vorticity flux divergence. In Eq. (6), the absolute vorticity advection [(a) plus (b) plus (c)] is interpreted as the response to the forcing [(d) plus (e) plus (f)].

Figure 13 shows each term of Eq. (6) at $40^{\circ} \mathrm{N}$ for $\mathrm{H} 0$. Zonal vorticity advection (Fig. 13a) is positive above the downslope of the mountain and negative over the ocean, in agreement with the negative (positive) vorticity around $90^{\circ} \mathrm{E}\left(90^{\circ} \mathrm{W}\right)$ in the upper troposphere (Fig. 7b). Meridional vorticity advection (Fig. 13b) and the beta term (Fig. 13c) are less than half of zonal vorticity advection. The vortex-tube stretching [the term (d) in Eq. (6)] is positive around $120^{\circ} \mathrm{E}$ and $300 \mathrm{hPa}$ and negative around $120^{\circ} \mathrm{W}$ and $300 \mathrm{hPa}$ (Fig. 13d), which balances positive zonal vorticity advection. Vorticity ad- 


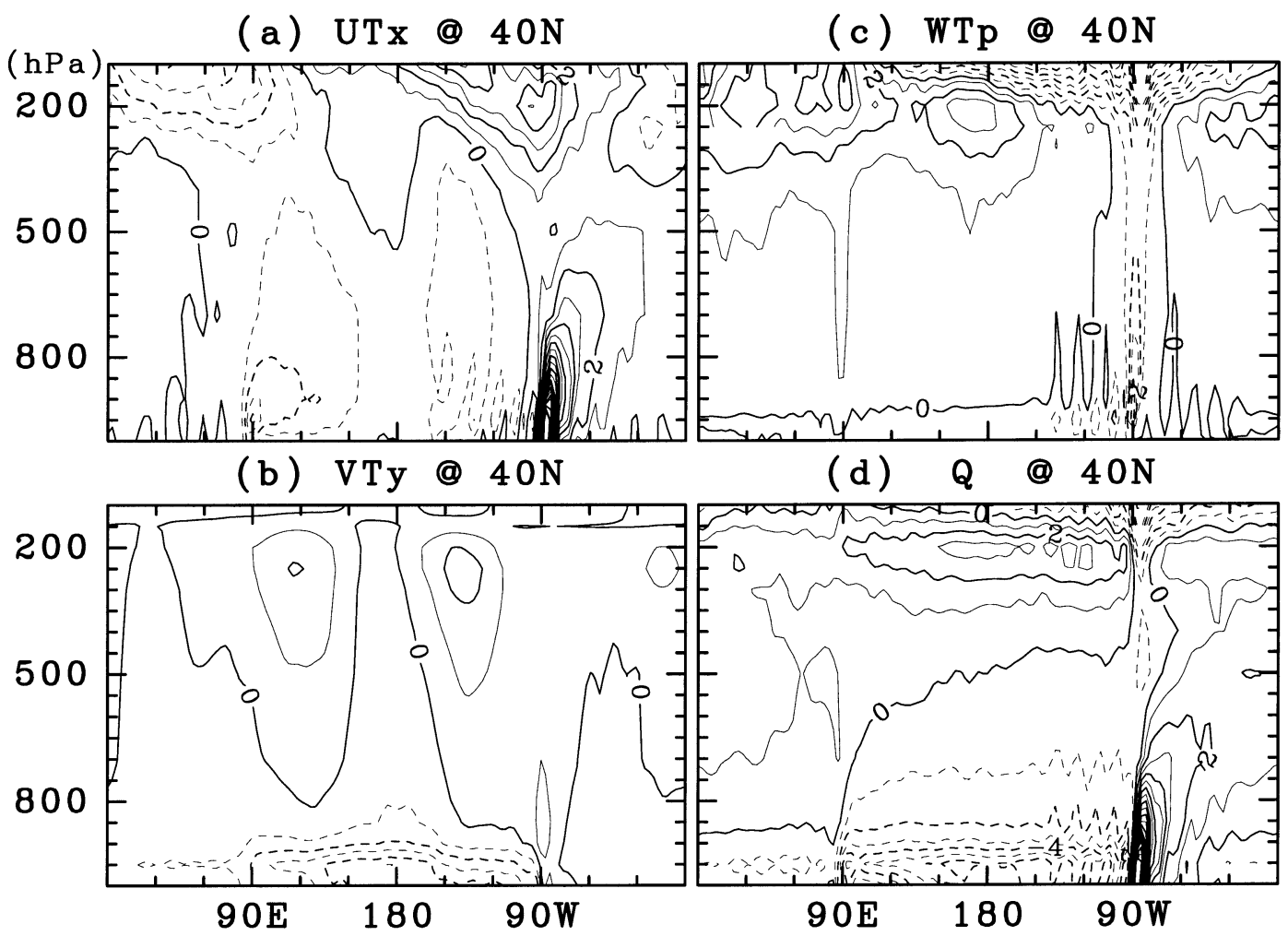

FIG. 12. Vertical cross sections for (a) zonal, (b) meridional, and (c) vertical temperature advection, and (d) timemean diabatic heating in the time-mean thermodynamical equation at $40^{\circ} \mathrm{N}$ for I0. These correspond to the terms (a), (b), (c), and (d) in Eq. (5), respectively. Contour interval is $1 \mathrm{~K}^{-1 a y}{ }^{-1}$; negative contours are dashed.

VORTICITY BUDGET ANALYSIS $40 \mathrm{~N}$ HO

(a) ZONAL ADV $u_{\psi} \xi_{\mathbf{x}}$

(d) STRETCH $(f+\zeta) D$

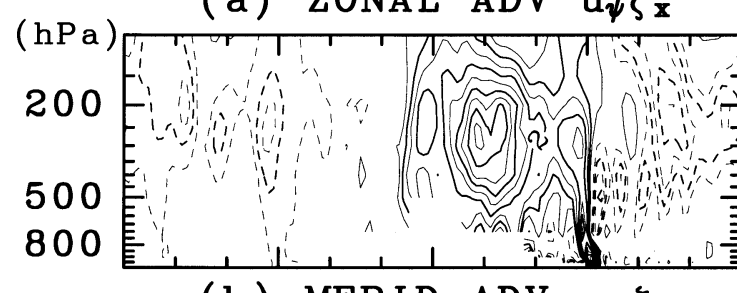

(b) MERID ADV $\mathrm{v}_{\psi} \zeta_{\mathrm{y}}$
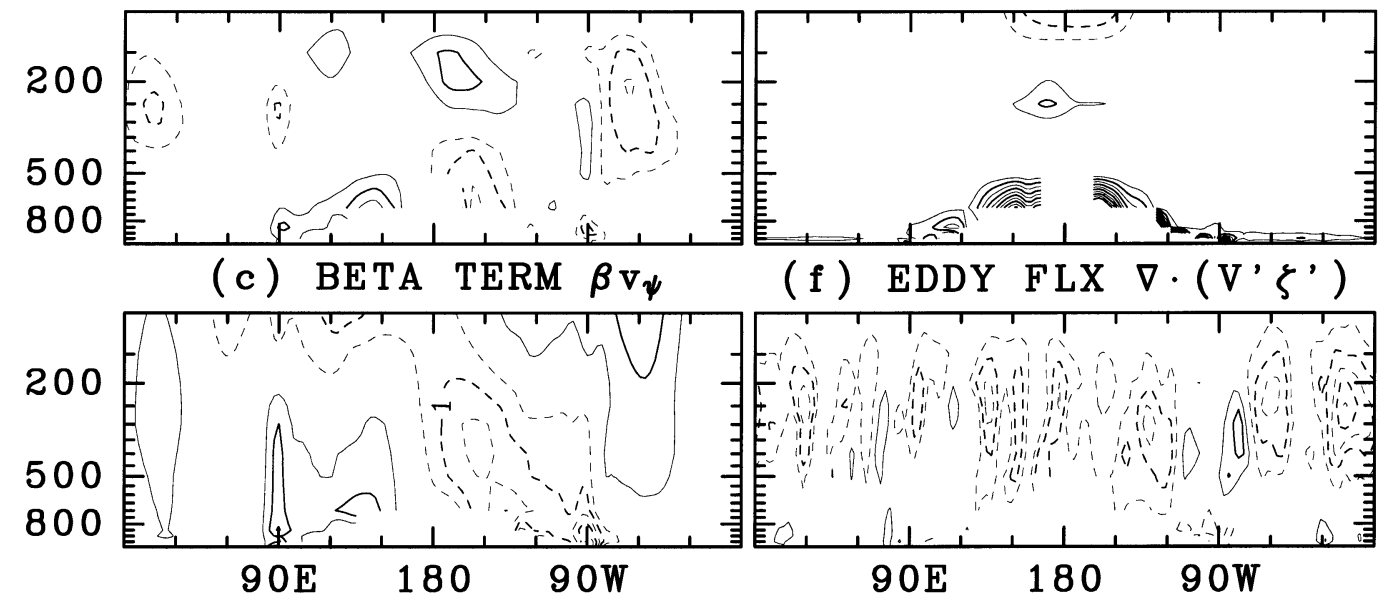


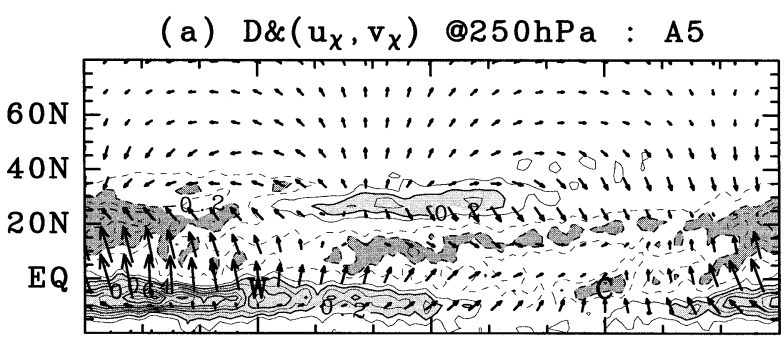

(b) $\mathrm{D} \&\left(\mathrm{u}_{\chi}, \mathrm{v}_{\chi}\right) @ 850 \mathrm{hPa}: \mathrm{A} 5$

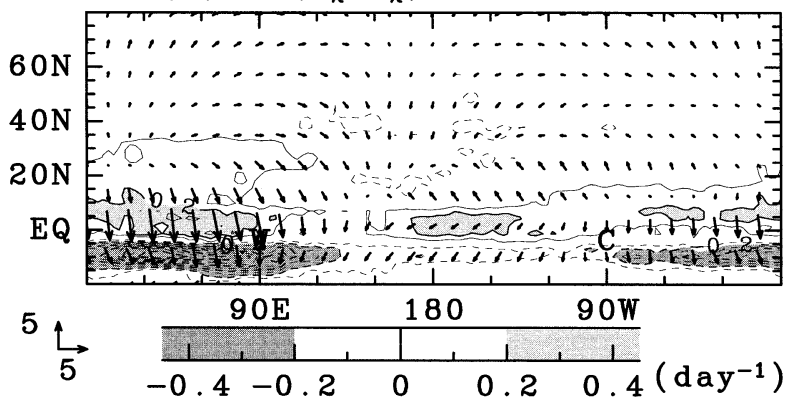

FIG. 14. Divergence (contours and shading) and divergent wind (vectors) for $\mathrm{A} 5$ at (a) $250 \mathrm{hPa}$ and (b) $850 \mathrm{hPa}$. Contour intervals are 0.1 day $^{-1}$ with negative contours dashed and zero contours removed. The reference arrows in the bottom left corner show the north and east components of a $5 \mathrm{~m} \mathrm{~s}^{-1}$ wind.

vection by divergent wind [the term (e) in Eq. (6)] is quite small except for the boundary layer (Fig. 13e). The eddy vorticity flux has negative values nearly everywhere at this latitude, with some small-scale variations (Fig. 13f). Therefore, upslope (downslope) of the mountain, the divergence (convergence) is associated with vortex tube squeezing (stretching), which is the effective forcing for generating extratropical stationary eddies in $\mathrm{H} 0$.

\section{Tropical forcing mechanism}

Tropical SSTs are most effective in generating subtropical stationary eddies and modulating the subtropical jet. In this section, we examine the formation mechanism of subtropical stationary eddies in the Aqua Planet experiments. We also investigate the dependence of the model response on the magnitude of the tropical SST forcing.

\section{a. Hadley circulation}

We first investigate the tropical divergence field induced by the zonal variation in SST. West to the warm water pool, strong divergence (convergence) exists in

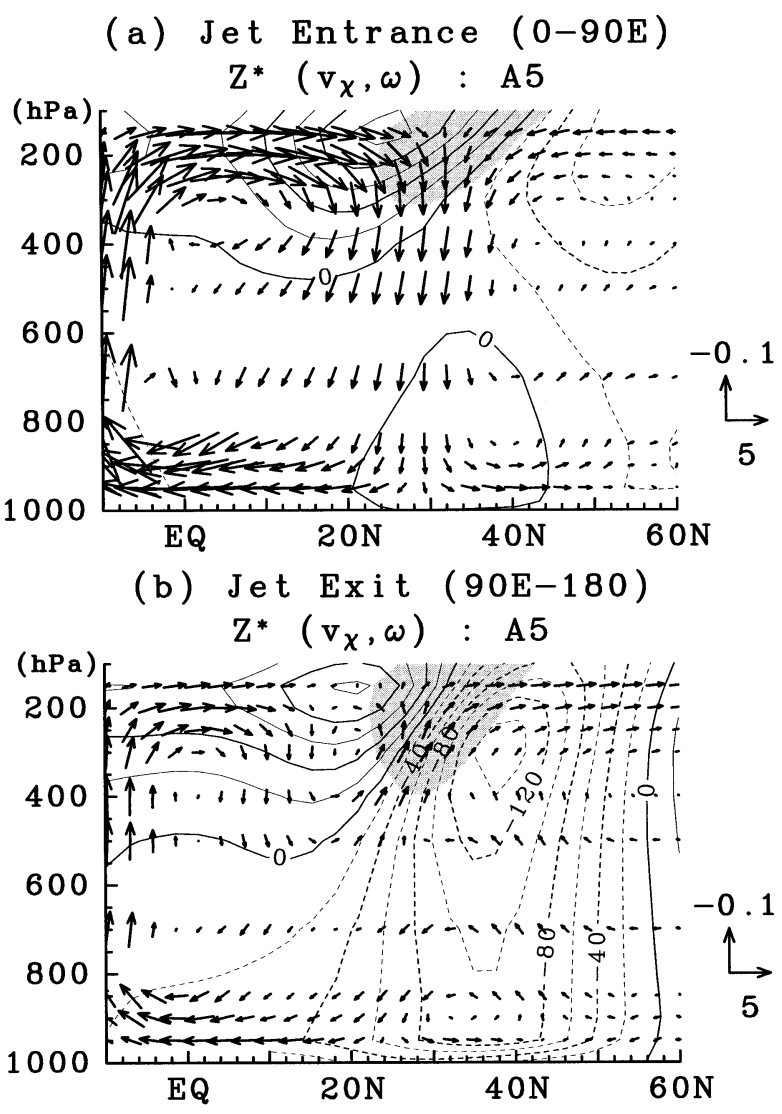

FIG. 15. Meridional cross sections for stationary eddy geopotential heights (contours) and meridional divergent wind and vertical velocity (arrows), averaged over (a) $0^{\circ}-90^{\circ} \mathrm{E}$, and (b) $90^{\circ} \mathrm{E}-180^{\circ}$ for A5. Contour intervals are $20 \mathrm{~m}$ with negative contours dashed. The reference arrows on the right are $5 \mathrm{~m} \mathrm{~s}^{-1}$ for meridional and -0.1 $\mathrm{hPa} \mathrm{s}^{-1}$ vertical velocity, respectively. Shading denotes westerly wind $>60 \mathrm{~m} \mathrm{~s}^{-1}$.

the upper (lower) troposphere in the Tropics (Figs. 14a and 14b), in contrast to the weak divergence over the cold water pool. Such tropical convection induces zonal asymmetry in the Hadley circulation, which is locally enhanced (suppressed) at the jet entrance (exit). These zonal variations in the local Hadley circulation force subtropical and extratropical stationary eddies.

Figure 15 displays the meridional component of divergent wind and vertical velocity (vectors), along with eddy geopotential height (contours). Subtropical westerlies blow between subtropical anticyclone and extratropical cyclone. At the jet entrance (upper panel), the upper branch of Hadley circulation reaches the axis of subtropical westerly jet. At the jet exit, in comparison,

Fig. 13. Vertical cross sections for (a) zonal and (b) meridional advection of time-mean relative vorticity, (c) planetary vorticity advection, (d) vortex-tube stretching, (e) vorticity advection by divergent wind, and (f) transient-eddy vorticity flux divergence at $40^{\circ} \mathrm{N}$ for $\mathrm{H} 0$. These are the terms (a), (b), .., and (f) in Eq. (5), respectively. Contour intervals are $0.5 \times 10^{-10} \mathrm{~s}^{-2}$ with negative contours dashed and zero contours removed. 


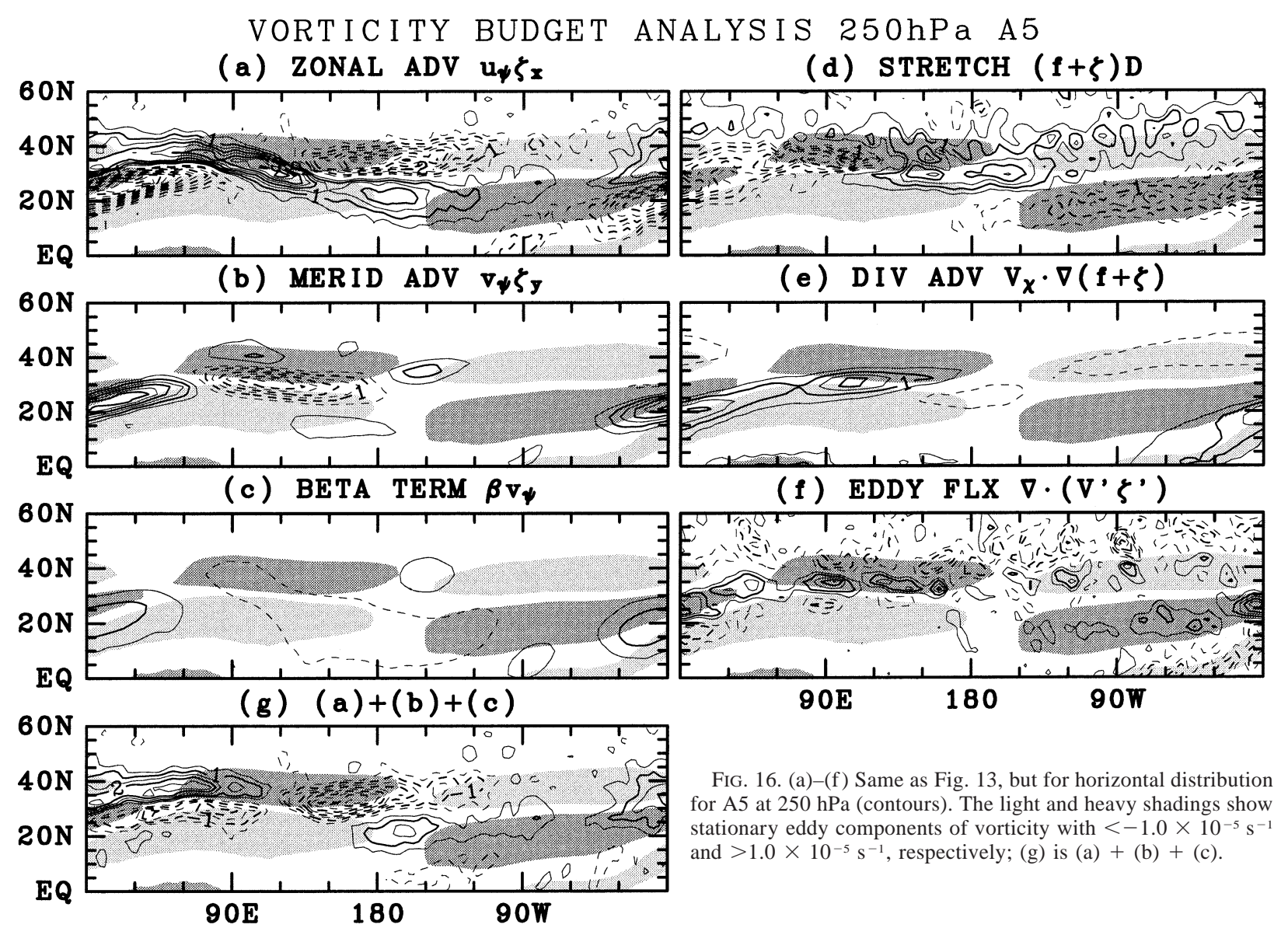

the Hadley circulation is much weaker and its downdraft is found at $17^{\circ} \mathrm{N}$, a latitude much to south of the jet axis.

\section{b. Vorticity budget analysis}

We perform the vorticity budget analysis for A5 to study how zonally asymmetric divergence induced by tropical deep convection affects subtropical stationary eddies.

Figure 16 shows the terms of Eq. (6) at $250 \mathrm{hPa}$ for A5. Zonal vorticity advection (Fig. 16a) has a quadrupole pattern around the jet core, in agreement with the positive (negative) vortex (shading in Fig. 16) north (south) to the jet core. Large meridional vorticity advection (Fig. 16b) south to the jet axis compensates the zonal vorticity advection. The beta effect (Fig. 16c) is quite small. The total absolute vorticity advection (Fig. $16 \mathrm{~g}$ ) has the same polarity as the zonal vorticity advection.

We now concentrate on the southwest of the jet core (around $30^{\circ} \mathrm{E}$ and $25^{\circ} \mathrm{N}$ ) where strong northward divergent winds accompanied with tropical heating prevail. In this region, the total absolute vorticity advection (Fig. 16g) is negative. The vorticity advection by divergent wind (Fig. 16e) is positive and tends to balance the total absolute vorticity advection, while vortex-tube stretching (Fig. 16d) and transient eddy vorticity flux divergence (Fig. 16f) are negative. Therefore the locally enhanced Hadley circulation to the west of the tropical warm water pool, which leads to vorticity advection by divergent wind, essentially forces subtropical stationary eddies.

\section{c. Nonlinearity}

Here we carry out a set of experiments $(\mathrm{A} 0, \mathrm{~A} 1, \ldots$, and A8 in Table 1) and examine the sensitivity of the model response to the amplitude of tropical SST forcing. As in section 3c, we will focus on the wavenumber-one component, analyzing its amplitude, phase, and standard deviation. For this analysis, we choose the following variables: zonal wind velocity along the subtropical jet axis ( $U$ in Fig. 17), relative vorticity in the center of subtropical stationary eddies $(\zeta)$, and tropical divergence $(D)$ and precipitation $(P)$.

The response to zonal precipitation variations is nonlinear in both the Tropics and extratropics. In the Tropics, while precipitation increases at an accelerated rate with SST, the rate of increase in tropical divergence slows down as SST increases (Fig. 17a). This nonlinear 
(a) $\operatorname{AMP} / \mathrm{SD}(\sigma)$

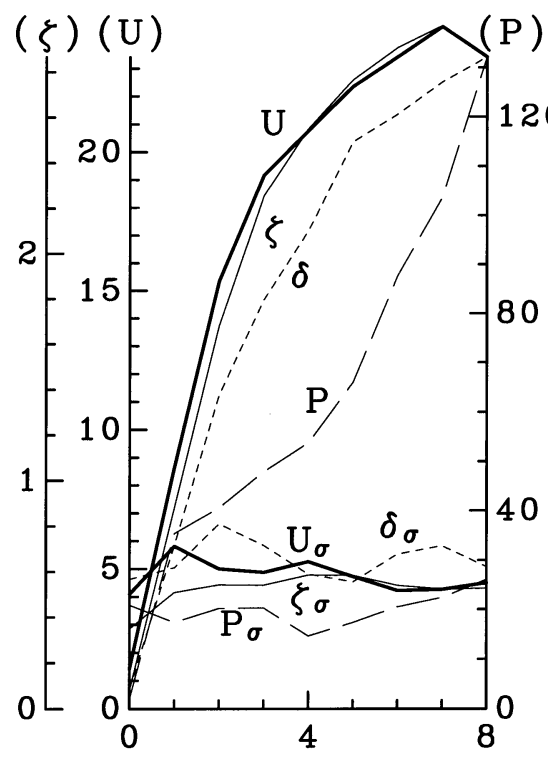

SST Variation $(K)$ (b) Phase
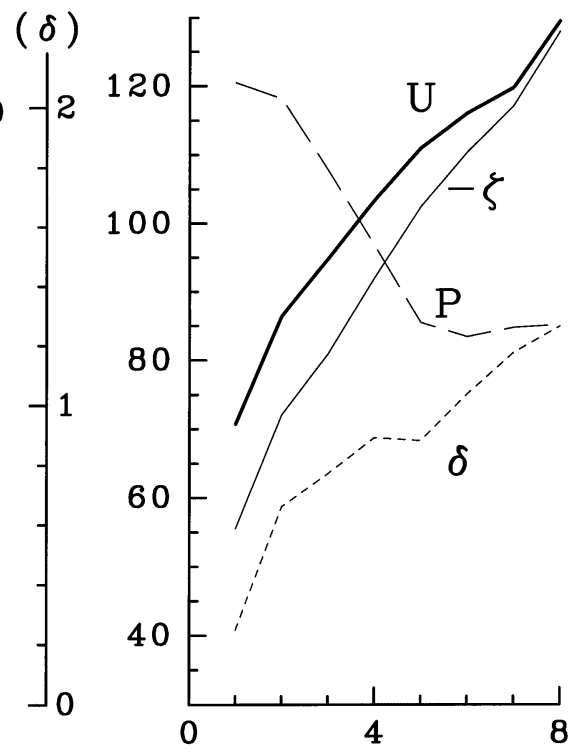

SST Variation $(K)$

FIG. 17. (a) The amplitude and (b) phase of several wavenumber-one components based upon 30-day-averaged data. Solid thick line denotes subtropical zonal velocity $U\left(\mathrm{~m} \mathrm{~s}^{-1}\right)$ averaged over $25^{\circ}-35^{\circ} \mathrm{N}$ and $200-300 \mathrm{hPa}$; solid thin line denotes vorticity $\zeta\left(10^{-5} \mathrm{~s}^{-1}\right)$ over $15^{\circ}-25^{\circ} \mathrm{N}$ and 200-300 hPa; line with long dashes, tropical rainfall $P\left(\mathrm{~W} \mathrm{~m}^{-2}\right)$ over $8^{\circ} \mathrm{S}-2^{\circ} \mathrm{N}$; line with short dashes, tropical divergent $\delta\left(10^{-6} \mathrm{~s}^{-1}\right)$ over $8^{\circ} \mathrm{S}-2^{\circ} \mathrm{N}$ and $200-300 \mathrm{hPa}$. The lines with a subscript $\sigma$ denotes the std dev of the fluctuation around the time mean. The phase is evaluated when the fluctuation is smaller than the amplitude in (a).

tropical divergence response may be due to the nonlinearity of the Clausius-Clayperon equation. Under the assumption that relative humidity remains more or less constant, specific humidity at the convection center, which is roughly collocated with maximum SST, increases with zonal SST variations exponentially. Since precipitation roughly equals the moisture convergence, tropical divergence should increase with SST forcing at a slower rate than precipitation.

The extratropical stationary wave response varies its amplitude somewhat in line with the tropical divergence, except between A7 and A8. In the phase diagram (Fig. 17b), the tropical precipitation center shifts westward with increasing SST forcing. By contrast, tropical divergence and extratropical stationary wave both shift their phase eastward (Hendon 1986). The extratropical

TABLE 2. Stationary eddy geopotential height amplitude $(\mathrm{m})$ of zonal wavenumber one, two, and three at $47^{\circ} \mathrm{N}$ and $250 \mathrm{hPa}$ for the control run (CTR), the CTR - NM, the NM - Tx0 differences, and the Tx0 run.

\begin{tabular}{lrcr}
\hline \hline & \multicolumn{3}{c}{ Zonal wavenumber } \\
& 1 & 2 & \multicolumn{1}{c}{3} \\
\hline CTR & 140.4 & 157.0 & 106.6 \\
CTR - NM & 90.9 & 105.0 & 34.7 \\
NM - Tx0 & 32.8 & 76.4 & 81.4 \\
Tx0 & 53.1 & 21.8 & 38.2 \\
\hline
\end{tabular}

response to tropical divergence is not exactly linear. In A2, both anticyclones and cyclones around the subtropical westerly jet are elongated in the east-west direction (Fig. 18a). On the other hand, in A8, a vortex pair in the subtropics not only shifts eastward but is also meridionally distorted with northeastward phase tilt (Fig. 18b). We therefore found that the subtropical response to tropical SST forcing shows some nonlinearity.

Tropical SST forcing also modifies zonal-mean field. As tropical forcing is strong, the zonal-mean zonal wind becomes westerly even in the Tropics and subtropical westerly jet speed falls down (Fig. 3b). Even though the stationary-eddy response in the subtropics could be described by the linear theory based upon a prescribed zonal-mean flow, the changes of zonal-mean zonal wind in the subtropics should be recognized as nonlinear atmospheric response to the tropical SST forcing (cf. Hoskins et al. 1999). In the future, we plan to use a linearized model based on this AGCM (Watanabe and Kimoto 2000) and compare its results with AGCM results to further study the extratropical response.

\section{Conclusions and further discussion}

In this paper, we have conducted a series of AGCM experiments with idealized boundary conditions to isolate the boundary forcings for the extratropical station- 


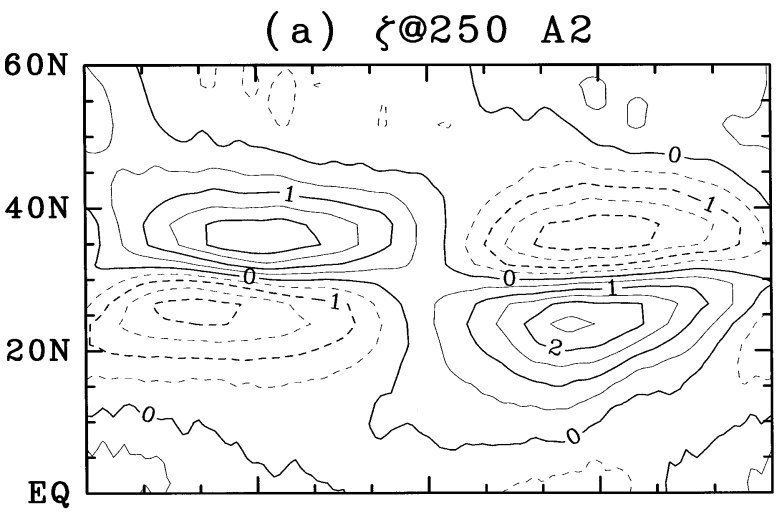

(b) $\zeta @ 250 \quad$ A8

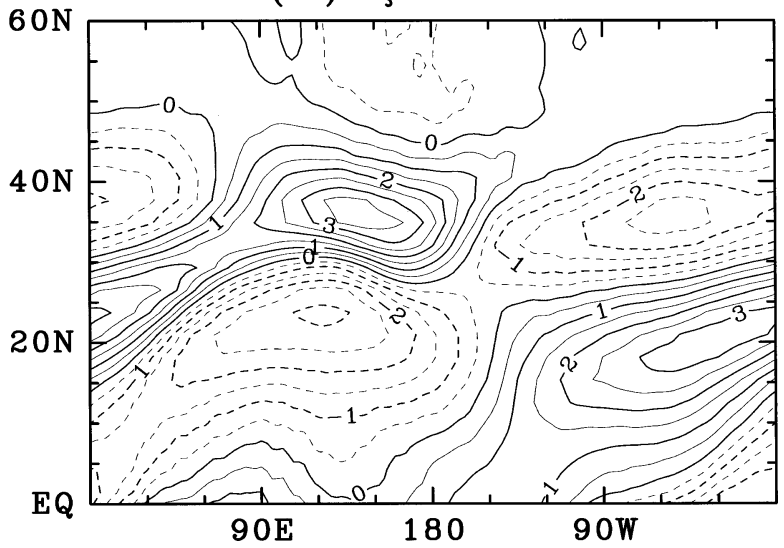

FIG. 18. Stationary eddy components of vorticity at $250 \mathrm{hPa}$ for (a) A2 and (b) A8. Contour intervals are $0.5 \times 10^{-5} \mathrm{~s}^{-1}$ with negative contours dashed.

ary eddies: extratropical land-sea contrast, large-scale mountains, and tropical SST variation. The major results are as follows.

1) Extratropical land-sea contrast, while forcing strong temperature variations in the lower atmosphere, induces only modest stationary waves in the upper troposphere in the high latitudes. Orographic-induced stationary eddies are robust and show strong vertical propagation into the stratosphere.

2) Zonal variations in tropical SST induce stationary waves both in the subtropics and midlatitudes. A robust jet core resides between the baroclinic subtropical and barotropic midlatitude waves. Locally enhanced Hadley circulation forms to the north of the tropical warm pool where strongest deep convection takes place. In the vorticity budget analysis, we found that this zonal variation in divergence leads to the formation of a subtropical anticyclone in the upper troposphere.

3) Zonal variations in tropical SST forcing also affect the zonal-mean circulation. With increasing tropical SST forcing, the Hadley circulation becomes slightly weaker and the westerlies occupy the equatorial upper troposphere.

4) The storm track induced by orography and by tropical SST forcing forms downstream of the subtropical jet core as in observations. By contrast, the extratropical land-sea distribution alone is not enough to form significant zonal asymmetry of storm track.

We have examined effects of three independent types of surface boundary forcing, namely, tropical SST, extratropical land-sea distribution and orography. Tropical SST variations are an obvious cause of zonal variations in tropical convection, which then force extratropical stationary eddies in the upper troposphere as discussed in sections $5 \mathrm{a}$ and $5 \mathrm{~b}$. In many previous studies, tropical convection is taken as an independent forcing for stationary eddies. The other two types of surface boundary forcings can also induce zonal changes in tropical precipitation, albeit to lesser degrees. Midlatitude orography forces Rossby waves that propagate into the Tropics and induce significant wave-mean flow interaction near the critical latitude. Indeed, in the Northern Hemisphere winter, wavetrains forced by Tibetan Plateau and Rocky Mountains penetrate into the tropical western Pacific and Atlantic, respectively, where they significantly contribute to zonal asymmetry in tropical convection (Nigam et al. 1988). This orographically forced zonal asymmetry in tropical convection in our H0 run (Fig. 11b) is much smaller than in A5 (Fig. 11c). The zonal variations in precipitation induced by the extratropical land-sea distribution is even smaller (Fig. 11a). It follows that extratropical surface boundary forcing is only a minor mechanism for tropical precipitation changes.

The formation of zonally localized storm track in our experiments is quite interesting. Off the east coast of a major continent, the cold air is in contact with the warm sea surface. The reduced static stability forms a localized maximum of baroclinicity there in the lower atmosphere (Hoskins and Valdes 1990). Such a region of high baroclinicity is indeed found off the east coast of the model continent. The rather zonally uniform distribution of midlatitude storm activity in I0 is somewhat surprising, suggesting that the observed Pacific and Atlantic storm tracks are probably not forced by the landsea distribution. In our model experiments, the storm track shows a tendency to form downstream of the westerly jet core, suggesting that it is sensitive to deep vertical wind shear in support of Hoskins and Valdes (1990). Extratropical orography is particularly effective in forming localized storm track in our model as indicated by Chang and Orlanski (1993). Whitaker and Dole (1995) suggested that the storm track is terminated by barotropic decay process associated with zonal variations in baroclinicity and the deformation of the basic state. The latter can result from stationary waves induced by large-scale orography. A more detailed discussion on storm tracks will be presented in our next study. 
( a )Z ( C TR-NM)@250hPa

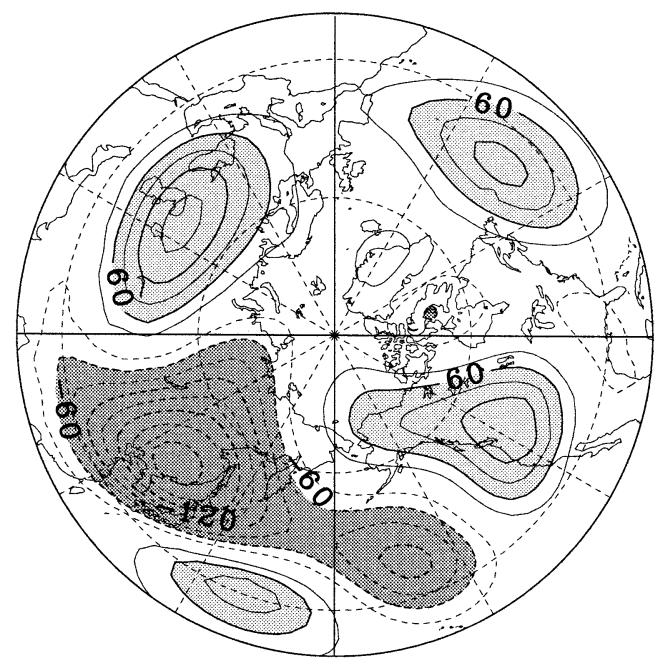

(c) Z ( Tx0)@250hPa

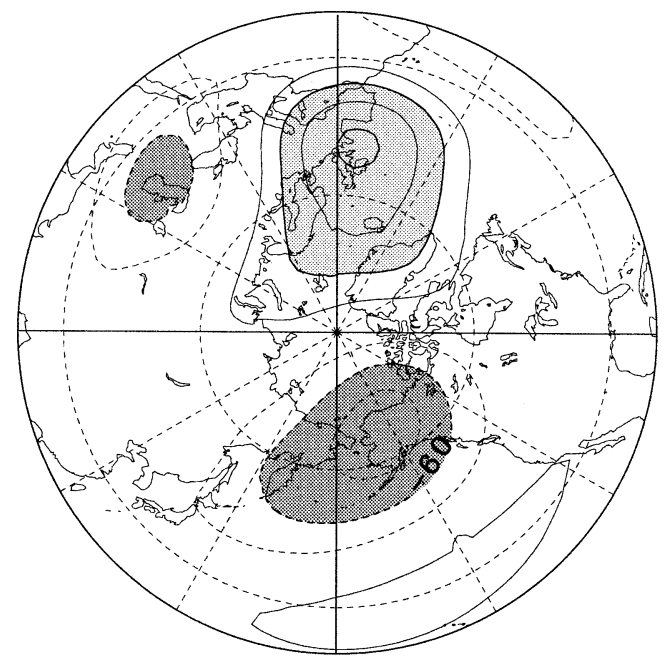

In addition to the idealized experiments, we have also carried out two additional simulations (Table 1) under more realistic conditions: A run with no mountains (NM) and one with zonally uniform SSTs (Tx0). Examination of the difference fields between these two runs and CTR (Fig. 1b) indicates the effect of various surface boundary forcing: CTR - NM for orography, NM - Tx0 for tropical SST, and Tx0 for land-sea distribution. The amplitudes of these difference fields in the midlatitudes are of the order of $100 \mathrm{~m}$ (Table 2). Figure 19 displays stationary eddies of $250-\mathrm{hPa}$ geopotential height for the Tx0 run (Fig. 19c), and the difference fields for CTR - NM (Fig. 19a) and NM - Tx0 (Fig. 19b). Stationary eddies induced by orography and zonal variations of SST are dominated by the zonal wavenumbers two and three, while those by land-sea contrast are of larger scales and

\section{( b ) Z ( NM-Tx0)@250hPa}

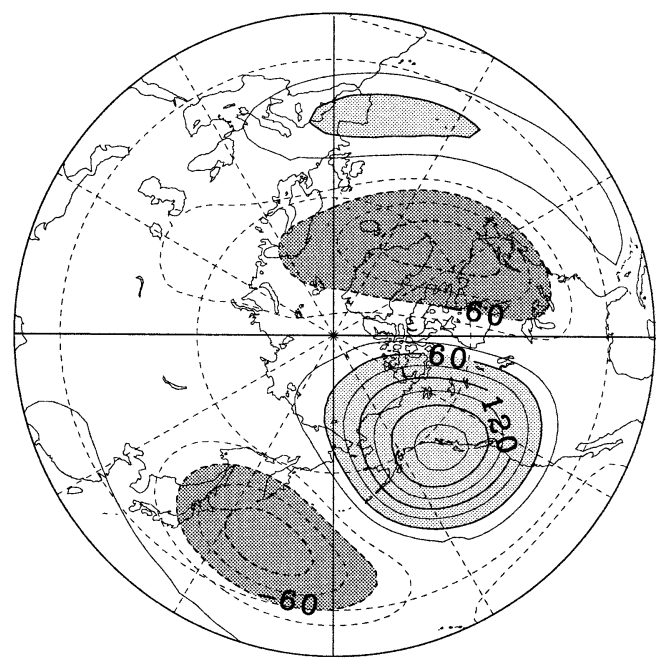

FIG. 19. Same as Fig. 1b, but for (a) CTR - NM, (b) $\mathrm{NM}-\mathrm{Tx} 0$, and (c) Tx0. have a strong wavenumber-one structure (Table 2). The East Asian-Pacific jet is maintained by an orography (SST) induced trough at its entrance (exit). The SSTforced anticyclone in the eastern Pacific has twice as large an amplitude as the orographically forced one. In the Atlantic, thermal forcing is more important than orographic. The cyclone over Hudson Bay is almost entirely caused by the SST forcing while the anticyclone over western Europe is by the land-sea contrast. In a broad sense, this result is consistent with previous AGCM and linear model studies (Held 1983; Blackmon et al. 1987; Valdes and Hoskins 1989). It is also similar to the results from idealized AGCM experiments presented in this paper: The cyclonic (anticyclonic) eddy over the western (eastern) Pacific (Fig. 19b) is similar to that in A5 (Fig. $4 c$ ); the cyclonic (anticyclonic) circulation on the east 
(west) coast of Eurasia corresponds to the response of the idealized land-sea distribution (Fig. 4a); the anticyclonic (cyclonic) circulation over the western (eastern) Eurasia resembles that in $\mathrm{H} 0$.

Acknowledgments. We thank A. Numaguti and M. Kimoto for permitting us to use CCSR/NIES AGCM version 5.4; K. Yamazaki, Y. Tanimoto, and S. Yoden for helpful discussion; and S. Nigam and two anonymous reviewers for comments that led to improved presentation. We dedicate this paper to A. Numaguti for his efforts in developing the CCSR/NIES AGCM version 5.4 and his generosity to make open his code to the Japanese university community. Supported by Grant-in-Aid for Scientific Research (C) (2) 12640417 and Scientific Research on Priority Areas (B) (2) 11219203 from the Ministry of Education, Culture, Sports, Science and Technology of Japan; the Center for Climate System Research of University of Tokyo; and U. S. National Science Foundation. The figures were produced with the GFD-DENNOU Library.

\section{REFERENCES}

Andrews, D. G., J. R. Holton, and C. B. Leovy, 1987: Middle Atmosphere Dynamics. Academic Press, 489 pp.

Blackmon, M. L., 1976: A climatological spectral study of the 500 $\mathrm{mb}$ geopotential height of the Northern Hemisphere. J. Atmos. Sci., 33, 1607-1623.

_ J. M. Wallace, N.-C. Lau, and S. L. Mullen, 1977: An observational study of the Northern Hemisphere wintertime circulation. J. Atmos. Sci., 34, 1040-1053.

— G. W. Branstator, and G. T. Bates, 1987: An analysis of equatorial Pacific sea surface temperature anomaly experiments in general circulation models with and without mountains. J. Atmos. Sci., 44, 1828-1844.

Chang, E. K. M., and I. Orlanski, 1993: On the dynamics of a storm track. J. Atmos. Sci., 50, 999-1015.

Charney, J. G., and A. Eliassen, 1949: A numerical method for predicting the perturbations of the midlatitude westerlies. Tellus, $\mathbf{1}$, $38-54$.

Edmon, H. J., B. J. Hoskins, and M. E. McIntyre, 1980: EliassenPalm cross-sections for the troposphere. J. Atmos. Sci., 37, 26002616.

Geisler, J. E., M. L. Blackmon, G. T. Bates, and S. Muñoz, 1985: Sensitivity of January climate response to the magnitude and position of equatorial Pacific sea surface temperature anomalies. J. Atmos. Sci., 42, 1037-1049.

Gill, A. E., 1980: Some simple solution for heat-induced tropical circulation. Quart. J. Roy. Meteor. Soc., 106, 447-462.

Held, I. M., 1983: Stationary and quasi-stationary eddies in the extratropical troposphere: Theory. Large-scale Dynamical Processes in the Atmosphere, B. J. Hoskins and R. Pearce, Eds., Academic Press, 127-168.

— , and A. Y. Hou, 1980: Nonlinear axially symmetric circulation in a nearly inviscid atmosphere. J. Atmos. Sci., 37, 513-533.

Hendon, H. H., 1986: The time-mean flow and variability in a nonlinear model of the atmosphere with tropical diabatic forcing. $J$. Atmos. Sci., 43, 72-88.

Horel, J. D., and J. M. Wallace, 1981: Planetary-scale atmospheric phenomena associated with the Southern Oscillation. Mon. Wea. Rev., 109, 813-829.

Hoskins, B. J., and D. J. Karoly, 1981: The steady linear response of a spherical atmosphere to thermal and orographic forcing. $J$. Atmos. Sci., 38, 1179-1196.

- and P. J. Valdes, 1990: On the existence of storm-tracks. $J$. Atmos. Sci., 47, 1854-1864.

— I. I. James, and G. H. White, 1983: The shape, propagation, and mean-flow interaction of large-scale weather systems. $J$. Atmos. Sci., 40, 1595-1612.

, R. Neale, M. Rodwell, and G.-Y. Yang, 1999: Aspects of the largescale tropical atmospheric circulation. Tellus, 51A-B, 33-44.

Huang, R.-H., and K. Gambo, 1982: The response of a hemispheric multi-level model atmosphere to forcing by topography and stationary heat sources (I) Forcing by topography. J. Meteor. Soc. Japan, 60, 78-92.

Inatsu, M., H. Mukougawa, and S.-P. Xie, 2000: Formation of subtropical westerly jet core in an idealized GCM without mountains. Geophys. Res. Lett., 27, 529-532.

Lau, N.-C., 1979: The observed structure of tropospheric stationary waves and local balances of vorticity and heat. J. Atmos. Sci., 36, 996-1016.

Lindzen, R. S., and B. Farrell, 1980: A simple approximate result for the maximum growth rate of baroclinic instabilities. J. Atmos. Sci., 37, 1648-1654.

Manabe, S., and T. B. Terpstra, 1974: The effects of mountains on the general circulation of the atmosphere as identified by numerical experiments. J. Atmos. Sci., 31, 3-39.

Matsuno, T., 1966: Quasi-geostrophic motions in the equatorial area. J. Meteor. Soc. Japan, 44, 25-43.

Matthews, A. J., and G. N. Kiladis, 1999: Interaction between ENSO, transient circulation, and tropical convection over the Pacific. $J$. Climate, 12, 3062-3086.

Nigam, S., I. M. Held, and S. W. Lyons, 1986: Linear simulation of the stationary eddies in a general circulation model. Part I: The no-mountain model. J. Atmos. Sci., 43, 2944-2961.

,-- , and -1988 : Linear simulation of the stationary eddies in a general circulation model. Part II: The "mountain" model. J. Atmos. Sci., 45, 1433-1452.

Numaguti, A., 1999: Origin and recycling process of precipitating water over the Eurasian continent: Experiment using an atmospheric general circulation model. J. Geophys. Res., 104, 19571972.

_, M. Takahashi, T. Nakajima, and A. Sumi, 1997: Description of CCSR/NIES atmospheric general circulation model. CGER Supercomputer Monogr., No. 3, National Institute for Environmental Studies, Tsukuba, Japan, 1-48.

Plumb, R. A., 1985: On the three-dimensional propagation of stationary waves. J. Atmos. Sci., 42, 217-229.

Shen, X., M. Kimoto, and A. Sumi, 1998: Land-surface processes associated with interannual variability of broad-scale Asian monsoon simulated by the CCSR/NIES AGCM. J. Meteor. Soc. Japan, 76, 217-236.

Simmons, A. J., 1982: The forcing of stationary wave motion by tropical diabatic heating. Quart. J. Roy. Meteor. Soc., 108, 503534.

Smagorinsky, J., 1953: The dynamical influence of large-scale heat sources and sinks on the quasi-stationary mean motions of the atmosphere. Quart. J. Roy. Meteor. Soc., 79, 342-366.

Ting, M.-F., and I. M. Held, 1990: The stationary wave response to a tropical SST anomaly in an idealized GCM. J. Atmos. Sci., 47, 2546-2566.

Tokioka, T., and A. Noda, 1986: Effects of large-scale orography on January atmosphere circulation: A numerical experiment. J. Meteor. Soc. Japan, 64, 819-839.

Valdes, P. J., and B. J. Hoskins, 1989: Linear stationary wave simulation of the time-mean climatological flow. J. Atmos. Sci., 46, 2509-2527.

Wallace, J. M., 1983: The climatological mean stationary waves: Observational evidence. Large-Scale Dynamical Processes in 
the Atmosphere, B. J. Hoskins and R. Pearce, Eds., Academic Press, 27-53.

Watanabe, M., and M. Kimoto, 2000: Atmospheric-ocean thermal coupling in the North Atlantic: A positive feedback. Quart. J. Roy. Meteor. Soc., 126, 3343-3369.

,-- T. Nitta, and M. Kachi, 1999: A comparison of decadal climate oscillation in the North Atlantic detected in observed and a coupled GCM. J. Climate, 12, 2920-2940.
Whitaker, J. S., and R. M. Dole, 1995: Organization of storm tracks in zonally varying flows. J. Atmos. Sci., 52, 1178-1191.

Xie, S.-P., and K. Saito, 2001: Formation and variability of a northerly ITCZ in a hybrid coupled AGCM: Continental forcing and ocean-atmospheric feedback. J. Climate, 14, 1262-1276. 